\title{
Development and validation of a novel risk score to predict 5- year mortality in patients with acute myocardial infarction in China: a retrospective study
}

\author{
Yan Tang ${ }^{1}$, Yuanyuan Bai ${ }^{1}$, Yuanyuan Chen ${ }^{1}$, Xuejing Sun ${ }^{1}$, Yunmin Shi ${ }^{1}$, Tian He ${ }^{1}$, Mengqing Jiang ${ }^{1}$, Yujie \\ Wang ${ }^{1}$, Mingxing $\mathrm{Wu}^{2}$, Zhiliu Peng ${ }^{2}$, Suzhen Liu ${ }^{1}$, Weihong Jiang ${ }^{1}$, Yao Lu ${ }^{3}$, Hong Yuan ${ }^{1,3}$, Jingjing Cai ${ }^{\text {Corresp. } 1,3}$ \\ ${ }^{1}$ Department of Cardiology, The Third Xiangya Hospital, Central South University, Changsha, Hunan, China \\ 2 Department of Cardiology, Xiangtan Central Hospital, Xiangtan, Hunan, China \\ ${ }^{3}$ Center of Clinical Pharmacology, The Third Xiangya Hospital of Central South University, Changsha, Hunan, China \\ Corresponding Author: Jingjing Cai \\ Email address: caijingjing83@hotmail.com
}

Background. The disease burden from ischaemic heart disease remains heavy in the Chinese population. Traditional risk scores for estimating long-term mortality in patients with acute myocardial infarction (AMI) have been developed without sufficiently considering advances in interventional procedures and medication. The goal of this study was to develop a risk score comprising clinical parameters and intervention advances at hospital admission to assess 5-year mortality in AMI patients in a Chinese population.

Methods. We performed a retrospective observational study on $2722 \mathrm{AMI}$ patients between January 2013 and December 2017. Of these patients, 1471 patients from Changsha city, Hunan Province, China were assigned to the development cohort, and 1251 patients from Xiangtan city, Hunan Province, China, were assigned to the validation cohort. Forty-five candidate variables assessed at admission were screened using least absolute shrinkage and selection operator, stepwise backward regression, and Cox regression methods to construct the C2ABS2-GLPK score, which was graded and stratified using a nomogram and X-tile. The score was internally and externally validated. The C-statistic and Hosmer-Lemeshow test were used to assess discrimination and calibration, respectively. Results. From the 45 candidate variables obtained at admission, 10 potential predictors, namely, including Creatinine, experience of Cardiac arrest, Age, N-terminal Pro-Brain Natriuretic Peptide, a history of Stroke, Statins therapy, fasting blood Glucose, Left ventricular end-diastolic diameter, Percutaneous coronary intervention and Killip classification were identified as having a close association with 5-year mortality in patients with AMI and collectively termed the C2ABS2-GLPK score. The score had good discrimination (C-statistic $=0.811,95 \%$ confidence intervals $[\mathrm{Cl}], 0.786-0.836$ ) and calibration (calibration slope $=0.988$ ) in the development cohort. In the external validation 
cohort, the score performed well in both discrimination (C-statistic $=0.787,95 \% \mathrm{Cl}$, $0.756-0.818$ ) and calibration (calibration slope $=0.976$ ). The patients were stratified into low- ( $\leq 148)$, medium- (149 to 218 ) and high-risk ( $\geq 219)$ categories according to the C2ABS2-GLPK score. The predictive performance of the score was also validated in all subpopulations of both cohorts.Conclusion. The C2ABS2-GLPK score is a Chinese population-based risk assessment tool to predict 5-year mortality in AMI patients based on 10 variables that are routinely assessed at admission. This score can assist physicians in stratifying high-risk patients and optimizing emergency medical interventions to improve long-term survival in patients with AMI. 
1 Development and validation of a novel risk score to predict 5-year mortality in patients

2 with acute myocardial infarction in China: a retrospective study

4 Yan Tang ${ }^{1 *}$, Yuanyuan Bai ${ }^{*}$, Yuanyuan $\mathrm{Chen}^{1}$, Xuejing Sun ${ }^{1}$, Yunmin Shi ${ }^{1}$, Tian $\mathrm{He}^{1}$,

5 Mengqing Jiang ${ }^{1}$, Yujie Wang ${ }^{1}$, Mingxing $\mathrm{Wu}^{2}$, Zhiliu Peng ${ }^{2}$, Suzhen Liu ${ }^{1}$, Weihong Jiang ${ }^{1}$, Yao

6 Lu $^{3}$, Hong Yuan ${ }^{1,3}$, Jingjing Cai ${ }^{1,3}$

7

$8{ }^{1}$ Department of Cardiology, The Third Xiangya Hospital of Central South University, Changsha,

9 Hunan, China

$10{ }^{2}$ Department of Cardiology, Xiangtan Central Hospital, Xiangtan, Hunan, China

$11{ }^{3}$ Center of Clinical Pharmacology, The Third Xiangya Hospital of Central South University,

12 Changsha, Hunan, China

13

$14{ }^{*}$ These authors contributed equally to this work

16 Corresponding Author: 


\section{Jingjing Cai}

18138 Tong-Zi-Po Road, Changsha, Hunan, 410013, China

19 Email address: caijingjing83@hotmail.com 


\section{Abstract}

21 Background. The disease burden from ischaemic heart disease remains heavy in the Chinese

22 population. Traditional risk scores for estimating long-term mortality in patients with acute

23 myocardial infarction (AMI) have been developed without sufficiently considering advances in

24 interventional procedures and medication. The goal of this study was to develop a risk score

25 comprising clinical parameters and intervention advances at hospital admission to assess 5-year

26 mortality in AMI patients in a Chinese population.

27 Methods. We performed a retrospective observational study on 2722 AMI patients between

28 January 2013 and December 2017. Of these patients, 1471 patients from Changsha city, Hunan

29 Province, China were assigned to the development cohort, and 1251 patients from Xiangtan city,

30 Hunan Province, China, were assigned to the validation cohort. Forty-five candidate variables

31 assessed at admission were screened using least absolute shrinkage and selection operator,

32 stepwise backward regression, and Cox regression methods to construct the C2ABS2-GLPK

33 score, which was graded and stratified using a nomogram and X-tile. The score was internally

34 and externally validated. The C-statistic and Hosmer-Lemeshow test were used to assess

35 discrimination and calibration, respectively.

36 Results. From the 45 candidate variables obtained at admission, 10 potential predictors, namely,

37 including Creatinine, experience of Cardiac arrest, Age, N-terminal Pro-Brain Natriuretic

38 Peptide, a history of Stroke, Statins therapy, fasting blood Glucose, Left ventricular end-diastolic 
39 diameter, Percutaneous coronary intervention and Killip classification were identified as having

40 a close association with 5-year mortality in patients with AMI and collectively termed the

41 C2ABS2-GLPK score. The score had good discrimination (C-statistic $=0.811,95 \%$ confidence

42 intervals $[\mathrm{CI}], 0.786-0.836$ ) and calibration (calibration slope $=0.988$ ) in the development cohort.

43 In the external validation cohort, the score performed well in both discrimination (C-

44 statistic $=0.787,95 \%$ CI, 0.756-0.818) and calibration (calibration slope $=0.976$ ). The patients

45 were stratified into low- $(\leq 148)$, medium- (149 to 218$)$ and high-risk $(\geq 219)$ categories according

46 to the C2ABS2-GLPK score. The predictive performance of the score was also validated in all

47 subpopulations of both cohorts.

48 Conclusion. The $\mathrm{C} 2 \mathrm{ABS} 2-\mathrm{GLPK}$ score is a Chinese population-based risk assessment tool to

49 predict 5-year mortality in AMI patients based on 10 variables that are routinely assessed at

50 admission. This score can assist physicians in stratifying high-risk patients and optimizing

51 emergency medical interventions to improve long-term survival in patients with AMI. 


\section{Introduction}

53 Acute myocardial infarction (AMI) is the leading cause of mortality and morbidity worldwide.

54 However, the mortality rate attributed to AMI remains variable across different countries (Khan

55 et al., 2010; Fox et al., 2010; Roth et al., 2020). With the introduction of new treatments and

56 assessment tools over the past several decades, the overall mortality of AMI has been declining

57 in the Western countries (Rogers et al., 2008; Yeh et al., 2010; Laribi et al., 2014;Reed et al.,

58 2017), However, in China, the long-term mortality of AMI has not significantly decreased, and

the 5-year mortality rate is approximately $20 \%$ of 5-year mortality (Fox et al., 2010; Khan et al.,

60 2010; Yeh et al., 2010; Li et al., 2015;Chang et al., 2017; Szummer et al., 2019), even though the

61 1-year mortality of AMI patients in China has markedly decreased from approximately $22 \%$ in

621995 to $11 \%$ by 2014 (Fox et al., 2010; Roth et al., 2020). Moreover, by 2030, the number of

63 AMI patients in China is expected to reach 23 million which might translate to higher long-term

64 mortality (Song et al., 2020).

65 The Global Registry of Acute Coronary Events (GRACE) score is a widely used tool in

66 clinical practice for the prediction of mortality for up to 5 years in patients with acute coronary

67 syndrome (ACS) (Kozieradzka et al., 2011; Zdanyte et al., 2020). However, the GRACE score

68 was established based on clinical parameters at hospital admission, without consideration of

69 revascularization intervention and medication advances in recent years. Furthermore,

70 contemporary risk prediction scores such as the Korea Acute Myocardial Infarction Registry 
71 (KAMIR) score based on the Korean population have mainly focused on the factors assessed at

72 hospital discharge, which may not be beneficial for optimizing emergency care and in-hospital

73 treatments for patients with AMI (Kim et al., 2011).

74 Therefore, we developed and validated a risk assessment score that integrates clinical

75 variables at hospital admission and advances in interventional procedures and medication to

76 predict 5-year mortality in patients with AMI. This tool will assist physicians in identifying

77 patients who are at high risk of long-term mortality and thus optimize the medical treatments to 78 improve the survival of AMI patients in China.

Materials \& Methods

81 Study Populations and Procedure

82 We conducted a retrospective observational study of 3088 inpatients with International

83 Classification of Disease [ICD] Version 9 diagnosed AMI based on the results of the coronary

84 angiography in Hunan Province, China. Only patients with type 1 AMI, defined as plaque-

85 mediated culprit lesions, were included in our study. The exclusion criteria were age younger

86 than 18 years or older than 90 years; the length of hospital stay $<1$ day with a lack of information

87 about the entire hospitalization for AMI, including information on death; severe hepatic impairment (alanine transaminase $[\mathrm{ALT}]>400 \mathrm{U} / \mathrm{L}$ or total bilirubin [Tbil] $>340 \mu \mathrm{mol} / \mathrm{L}$ ); 
89 malignancy; life-threatening infection; severe renal impairment (estimated glomerular filtration

90 rate $[\mathrm{eGFR}]<15 \mathrm{~mL} / \mathrm{min}$ ); severe and active autoimmune disease or haemodynamically unstable

91 trauma at admission. This study was approved by the Ethics Committee of the Third Xiangya

92 Hospital of Central South University and Xiangtan Central Hospital (IRB approval numbers:

93 2019-S489 and 2020-11-001, respectively).

94 For the development cohort, 1723 patients with AMI who were admitted between January

952013 and December 2017 were consecutively recruited for this study from the Third Xiangya

96 Hospital of Central South University in Changsha city. The last date of follow-up for the

97 mortality was August 31, 2021. Patients with severe hepatic dysfunction ( $\mathrm{n}=58)$, severe renal

98 impairment $(\mathrm{n}=85)$, active malignancy $(\mathrm{n}=54)$, severe autoimmune disease $(\mathrm{n}=8)$, current life-

99 threatening infection $(n=39)$, and haemodynamically unstable trauma $(n=4)$ at admission as well

100 as those with a length of hospital stay $<1$ day $(n=4)$ were excluded. The remaining 1471 patients

101 were included in the final analysis of the development cohort. Among the patients in the final

102 analysis, 292 patients died, and 9 patients were censored during the follow-up period.

103 For the external validation cohort, 1365 patients with ICD-9-confirmed AMI who were

104 admitted between January 2013 and December 2017 were consecutively included from the

105 Xiangtan Central Hospital in Xiangtan city. The last date of the follow-up was August 31, 2021.

106 Patients with severe hepatic dysfunction $(n=1)$, severe renal impairment $(n=24)$, active

107 malignancy $(\mathrm{n}=16)$, autoimmune disease $(\mathrm{n}=5)$, and haemodynamically unstable trauma $(\mathrm{n}=2)$ at 
108 admission as well as those with a length of hospital stay $<1$ day $(n=66)$ were excluded. Thus,

1091251 patients in the external validation cohort were included in the final analysis. Among these

110 patients, 186 patients died, and 65 patients were censored during the follow-up period.

111 The demographics and clinical characteristics, medical history, laboratory tests, imaging

112 examinations, and in-hospital interventions were obtained from patients' electronic medical

113 records. Mortality and its associated causes were collected from the death registry reporting

114 system of the National Center for Disease Control and Prevention (CDC) and were further

115 confirmed through telephone follow-up. The clinical data for the construction of the predictive

116 models were based on the measurements at admission for both cohorts. The ethics committee of

117 each hospital approved the study protocols. The need to obtain informed consent from the

118 patients was waived by both ethics committees. The study complied with the requirements in the

119 Transparent Reporting of a Multivariable Prediction Model for Individual Prognosis or

120 Diagnosis (TRIPOD) statement (Table S1) (Moons et al., 2015). A flowchart of the model

121 development and validation process is shown in Fig. 1.

123 Variable Selection and Model Development

124 Forty-five candidate variables were identified from the clinical variables available at hospital

125 admission and a literature review and entered into the selection process (Table S2). Of these, 
126 candidate variables with less than $20 \%$ missing data were included in the study (Table S3). The

127 variables selected for the predictive risk model were chosen from a total of the forty-five

128 candidate baseline parameters in a two-round process.

129 Least absolute shrinkage and selection operator (LASSO) regression was applied in the first-

130 round of variable selection in the development cohort. LASSO regression is capable of

131 minimizing the potential collinearity of variables and overfitting with the minimum lambda $(\lambda=$

132 0.037), and the mean squared error (MSE) is within one standard error of minimal MSE using

133 ten cross-validations (Tibshirani, 1997; McClelland et al., 2015). Eighteen variables were

134 selected from the first-round variable selection and entered into the second-round variable

135 selection. To further validate the variable selection, we repeated the random forest algorithm 100

136 times in the development cohort. The variables were evaluated by the importance and the out-of-

137 bag (OOB) value (Kruppa J et al.,2014; Speiser JL \& Durkalski VL \& Lee WM,2015).

138 The second round of variable selection was used to further select variables by multivariate

139 stepwise backward regression model. Five iterative processes were applied to develop a

140 parsimonious model while minimizing overfitting. An Akaike information criterion (AIC) value

$141<4.6$ and a $p$-value $>0.05$ were the cut-off values for the chosen final variables. Ten variables

142 were selected from the second round of variable selection. 
143 In the final model building stage, ten variables selected from the second-round of variable

144 selection were utilized in a multivariate Cox hazards regression model to develop an algorithm

145 for predicting 5-year all-cause mortality in AMI patients. Due to the unknown individual

146 absolute survival rate, we could obtain only the relative risks of AMI patients compared with

147 those without any risk factors based on the regression coefficients of all variables (Kalbfleisch

148 JD \& Prentice RL, 2002; Royston P\& Altman DG, 2013). To improve the application of the

149 C2ABS2-GLPK score in clinical practice, we transformed the Cox model into a nomogram

150 model. Every selected variable was given a continuous score from 0 to 100 corresponding to the

151 regression coefficient. The total risk score incorporating the ten predictors corresponded to the

152 relative survival rate.

153 The X-tile program was used to identify the optimal cut-off value of the risk score to stratify

154 patients with various degrees of mortality risk in the development cohort. The optimal cut-off

155 values and patient groups were based on the minimal $p$-value (0.003) and the maximum of chi-

156 square log-rank value ( $p$-value $<0.001)$ according to 5-year mortality in patients with AMI. The

157 optimal cut-off values were also validated in the external validation cohort (log-rank $p$ -

158 value $<0.001)($ Camp, Dolled-Filhart \& Rimm, 2004).

159 X-tile analysis revealed that patients with C2ABS2-GLPK scores $\leq 148$ points were stratified

160 into a low-risk group with a 5-year mortality of 6.6\%; Patients with C2ABS2-GLPK scores

161 between 149 and 218 points were graded as medium-risk with a 5-year mortality of 15.2\%; and 
162 patients with C2ABS2-GLPK scores $\geq 219$ points were categorized into a high-risk group with a

163 5-year mortality of 37.3\%. Moreover, based on our literature review, the predicted mortality in

164 each risk subgroup was similar to that of the GRACE score, thrombolysis in myocardial

165 infarction (TIMI) risk score and modified GRACE score during a follow-up period of more than

1665 years (Fox KA et al., 2010; Hall M et al., 2018;Kozieradzka A et al.,2011; Henderson RA et al., 167 2015).

169 Evaluation of Model Performance

170 The performance of the risk prediction model was evaluated by its discrimination and calibration.

171 Internal validation was performed in the primary development cohort. Given the censoring in the

172 development and external validation cohorts (Hippisley-Cox J \& Coupland C \& Brindle P, 2013;

173 Kalderstam J et al., 2013), 1000-times bootstrapped C-statistics were applied to assess the

174 discrimination of the risk prediction model in the development and external validation cohorts.

175 Calibration measures how accurately a model's predictions match the overall observed

176 mortality in the follow-up period. We equally split the observed 5-year mortality into equal

177 deciles across the development and external validation datasets. Calibration performance was

178 assessed with a calibration plot and summarized across the full range of mortality deciles using

179 the Hosmer-Lemeshow statistic. The calibration plot is generated from the means and standard 
180 deviation (SD) of the calibration slope and intercept. Furthermore, calibration of the C2ABS2-

181 GLPK score was measured by calibration-in-the-large, which is capable of reporting the

182 difference between the overall observed 5-year mortality and the mean predicted mortality in the 183 development cohort and external validation cohort.

\section{Missing Data Imputation,Cross Validation and Sensitivity Analyses}

Imputation for missing variables was considered if fewer than $20 \%$ of values were missing.

187 Multiple imputations were implemented to handle missing data with the "mice" package,which

188 is a widely accepted method for population datasets (Azur et al., 2011). Continuous and categorical data were imputed in a 10 sets with a 10 -iteration process based on the fitted conditional models until a stopping criterion was satisfied.

191 Missing data patterns were analysed by the "finalfit" package, and data were considered to be

192 missing at random, which means that each variable in the dataset is equally and randomly likely

193 to be missing and that the conditional likelihood of a missing value is partly dependent on other

194 missing variables, defined by the observed data. Missing at random is in contrast to missing

195 completely at random (Knight et al., 2020).

196 The linear regression model for continuous variables (mean error rate $15.4 \%, 95 \%$ CI, $12.3 \%$ -

$19716.7 \%$ ), logistic regression model for binary variables (mean error rate $6.1 \%, 95 \% \mathrm{CI}, 5.5 \%$ - 
$1987.8 \%$ ) and polytomous logistic regression for categorical variables (mean error rate 7.3\%, 95\%

199 CI, 6.5\%-9.2\%) with more than two levels were applied to the missing parameters to estimate the

200 imputation errors and internally cross-validated errors (Waljee et al., 2013; Knight et al., 2020).

201 To address the potential effect of missing data imputation, complete case data excluding

202 missing values were analysed to assess the robustness of the C2ABS2-GLPK model to predict 5-

203 year all-cause mortality. Thus, we performed sensitivity analyses by using complete case data to

204 assess the discrimination of the C2ABS2-GLPK model by C-statistics with 1000 iterations of

205 bootstrapping in the development and external validation cohorts, and to compare the results

206 with those of the imputed datasets.

207

208 Comparison with Existing Prognostic Scores

209 The performance of the C2ABS2-GLPK score was compared with that of existing prognostic

210 scores, including the GRACE score, KAMIR score and China Acute Myocardial Infarction

211 (CAMI) score, in the development and the external validation cohorts. The discrimination of the

212 GRACE score, KAMIR score and CAMI score was conducted in the samples with 1000

213 iterations of bootstrapping for both cohorts. Differences in the C-statistics were assessed using

214 the DeLong test (Harrell FE \& Lee KL\& Mark DB, 1996; Steyerberg et al., 2010). Moreover,

215 the bootstrapped discrimination of the C2ABS2-GLPK score was also evaluated in the subgroups 
216 based on sex, smoking statuses, ST segment elevation myocardial infarction (STEMI), type 2

217 diabetes, dyslipidaemia (low-density lipoprotein cholesterol [LDL-C] $>2.59 \mathrm{mmol} / \mathrm{L}$ ), or obesity

218 (body mass index $[\mathrm{BMI}] \geq 24$ ) in the development and external validation cohorts.

219 To evaluate the net benefits of the C2ABS2-GLPK score at the threshold probability for 5-

220 year mortality in the development and external validation sets, we also performed a decision

221 curve analysis to make a clinical judgement about the relative values of mortality (predicting a

222 true positive) and survival rate (predicting a false positive) associated with clinical prediction

223 scores (Vickers \& Elkin, 2006). The standardized net benefit was plotted against the threshold

224 probability of considering a patient at high risk of 5-year mortality for the C2ABS2-GLPK score

225 alone and the existing GRACE, KAMIR and CAMI scores applicable to more than 50\% of the

226 Chinese AMI patients in the external validation cohort.

228 Statistical Analysis

229 Group comparisons were conducted using the chi-squared test for equal proportions or a $\mathrm{t}$

230 tests for normally distributed data; otherwise, the Wilcoxon rank sum tests was used. A $p<0.05$

231 was considered indicative of statistical significance. Statistical analysis was performed using R

232 software (version 3.6.2, R Foundation). 


\section{Results}

\section{Patients Characteristics in the Development Cohort}

236 A total of 1471 patients with ICD-9-confirmed AMI between January 2013 and December 2017

237 from the Third Xiangya Hospital, Changsha city, Hunan Province, were included in the

238 development cohort. During a median follow-up of 5 years (range: 0.08 to 8.75 years), 292

239 patients (19.9\%) died, and $9(0.6 \%)$ were lost to follow-up before the end of the follow-up period.

240 The median age was 64 years (interquartile range (IQR), 55-72), and 71.4\% of the patients were

241 men. A total of $173(11.8 \%)$ patients had a history of stroke, 35 (2.4\%) experienced

242 preadmission or at-admission cardiac arrest, $844(57.4 \%)$ underwent immediate percutaneous

243 transluminal coronary intervention [PCI], 1457 (99.0\%) were administered statins at admission,

244 and 87 (5.9\%) developed acute heart failure ranked as Killip IV at admission. The median N-

245 terminal pro-brain natriuretic peptide [NT-proBNP] level at admission was $1639 \mathrm{pg} / \mathrm{ml}$ (IQR

246 611-3009), the median serum creatinine at admission was $82 \mu \mathrm{mol} / \mathrm{L}$ (IQR, 68-99), and the

247 median left ventricular end-diastolic diameter at admission was $49 \mathrm{~mm}$ (IQR, 45-51). The

248 baseline characteristics and their differences among the survivors, the deceased patients and the

249 censored patients in the cohort are shown in Table 1. 
251 Variable Selection and the C2ABS2-GLPK Model Establishment

252 Forty-five candidate clinical variables at admission from the patients in the development cohort

253 were included for variable selection. Among these variables, 18 variables were selected in the

254 first-round LASSO regression (Fig. S1, Table S4). The variables with an average OOB value >

$25520 \%$ obtained by repeating the random forest algorithm were similar to the variables selected by

256 LASSO regression. The importance of variables, as assessed by the random forest algorithm and

257 reported as the percent increase in MSE (\%IncMSE), is shown in Fig. S2 and Table S5. After

258 five rounds of multivariable stepwise backward regression, the 18 LASSO-selected variables

259 were reduced to 10 variables to enter into the final multivariable Cox proportional hazards model

260 (Table S6) and to construct the C2ABS2-GLPK model. The final 10 risk factors, namely,

261 admission or perihospital cardiac arrest, a history of stroke, Killip classification II-IV, fasting

262 blood glucose $\geq 10 \mathrm{mmol} / \mathrm{L}$, left ventricular end-diastolic diameter (LVDd) $\geq 60 \mathrm{~mm}$, age $\geq 70$,

263 serum creatinine $\leq 35 \mu \mathrm{mol}, \mathrm{NT}-\mathrm{proBNP} \geq 300 \mathrm{pg} / \mathrm{ml}$, absence of immediate statin

264 administration or PCI within $24 \mathrm{~h}$ after symptom onset, were incorporated into the risk score

265 model for the prediction of 5-year mortality in AMI patients (Table 2).

266

267 Construction of the Prognostic Nomogram and C2ABS2-GLPK Score 
268 The nomogram that incorporated the final 10 significant independent factors for predicting the 5-

269 year survival rate in the development cohort was established according to their regression

270 coefficients from the C2ABS2-GLPK model (Fig. 2).

271 Based on the nomogram, every selected variable was given a continuous score from 0 to 100

272 corresponding to the regression coefficient. The total C2ABS2-GLPK score was the sum of the

273 scores of each variable. The vertical line was dropped down from the total score row to estimate

274 the relative 1-, 2-, 3-, 4-, and 5-year all-cause survival rates for better application in clinical

275 practice (Fig. 2). The details of the score for each variable are shown in Table S7.

276

277

The Performance of the C2ABS2-GLPK Model and Score in the Development Cohort and

278

\section{Internal Validation}

In the development cohort, the C2ABS2-GLPK model showed good discrimination in predicting

5-year all-cause mortality in patients with AMI, with a C-statistic equal to $0.811(95 \%$ CI, 0.786-

281

0.836) (Table S8). Using the Hosmer-Lemeshow goodness-of-fit test, we found that this model

282

was well calibrated with good coherence between observed and predicted mortality across

283

deciles with the a Hosmer-Lemeshow statistic index of $5.277(p=0.728)$ (Table S8, Fig. 3). The

calibration slope was 0.988 , and the intercept was 0.716 in the prediction of 5-year mortality 
286 dataset indicated that the C2ABS2-GLPK model was perfect; however, the intercept was

287 typically far from 0.0 , suggesting that the predicted risk was an overestimation of 5-year

288 mortality in AMI patients. Nevertheless, the overall observed mortality versus the mean

289 predicted mortality for the C2ABS2-GLPK score were similar (calibration-in-the-large=0.02)

290 (Fig. 3).

291 According to the C2ABS2-GLPK score, AMI patients in the development cohort were

292 stratified into 3 distinct risk groups by the predicted mortality probabilities following the cut-off

293 points detected by the X-tile analysis. The predicted probability of dying within 5 years was

$2946.6 \%$ in the low-risk group (total points $\leq 148$ points), $15.2 \%$ in the medium-risk group (total

295 points, $149-218$ points), and $37.3 \%$ in the high-risk group (total points $>219$ points). Compared

296 to that in the low-risk group, the HRs (95\% CI) for the medium- and high-risk groups were 2.4

297 (95\% CI, 1.6-3.6) and 7.2 (95\% CI, 5.1-10.2), respectively (log-rank $p<0.001)$ (Fig. 2, Table 298 S10).

300 The Performance of the C2ABS2-GLPK Model and Score in the External Validation

301 Cohort

302 A total of 1251 patients with ICD-9-confirmed AMI between January 2013 and December 2017

303 from Xiangtan Central Hospital, Xiangtan city, Hunan Province, were included in the final 
304 external validation cohort (Table 1). During a median follow-up of 5 years (range: 0.08 to 8.58

305 years), 186 patients (14.9\%) died, and $65(5.2 \%)$ were lost to follow-up. The median age was 65

306 years (IQR, 56-73), and $72.8 \%$ of subjects were men. A total of $127(10.2 \%)$ patients had a

307 history of stroke, $38(3.0 \%)$ experienced preadmission and at-admission cardiac arrest, 867

308 (69.3\%) underwent immediate PCI, 1251 (100.0\%) were administered statins, and $66(5.3 \%)$

309 developed acute heart failure ranked as Killip IV at admission. The median NT-proBNP level

310 was $918 \mathrm{pg} / \mathrm{ml}$ (IQR, 213-2587), the median serum creatinine was $79 \mu \mathrm{mol} / \mathrm{L}$ (IQR, 67-95), and

311 the median left ventricular end-diastolic diameter at admission was $49 \mathrm{~mm}$ (IQR, 46-51).

312 The C-statistic of the C2ABS2-GLPK score, indicating its discrimination ability, was 0.787

$313(95 \%$ CI, 0.756-0.818) in predicting 5-year all-cause mortality for Chinese AMI patients in the

314 external validation cohort (Table S8). Calibration was also found to be excellent in the external

315 validation cohort: the Hosmer-Lemeshow statistic index for comparison of the observed and

316 predicted risk across deciles of 5-year all-cause mortality in the C2ABS2-GLPK model was

$3179.495(p=0.302)$ (Fig. 3, Table S8). The slope was 0.976 , and the intercept was 0.473 in the

318 prediction of 5-year mortality using the C2ABS2-GLPK model (Table S9). Although a slope

319 nearly equal to 1.0 in the external validation dataset suggested that the C2ABS2-GLPK model

320 was good, the intercept was well above 0.0 , indicating that the predicted risk was an

321 overestimation of 5-year mortality in AMI patients. Calibration was also found to be excellent 
322 for the C2ABS2-GLPK score: the overall observed mortality versus the mean predicted mortality

323 was nearly equal (calibration-in-the-large=0.03) (Fig. 3).

324 The C2ABS2-GLPK score was also applied to the external validation cohort. In the validation

325 cohort, AMI patients could still be divided into the same three risk categories according to the

326 total prediction score. The mean observed 5-year mortality rates in the low-, medium-, and high-

327 risk groups were $8.8 \%, 19.6 \%$, and $41.7 \%$, respectively. The Kaplan-Meier curves showed that

328 the HRs (95\% CIs) for the medium-risk and high-risk categories were $2.6(1.8-3.6)$ and 6.5 (4.6-

329 9.1), respectively, compared to that of the low-risk category (log-rank $p<0.001)$ (Fig. 2, Table

330 S10). The results indicated that a higher C2ABS2-GLPK score was associated with an increased

331 risk of 5-year mortality $(p<0.001)$.

332

333 Comparing the Performance of C2ABS2-GLPK Score with Existing Scores

334 To further validate the superiority in assessing the performance of C2ABS2-GLPK score, we

335 used the c-statistics to compare this score to the GRACE score, KAMIR score and CAMI score

336 in the development cohort and external validation cohort, respectively. The ability of each score

337 was assessed by c-statistic $(95 \% \mathrm{CI})$. Among them, the c-statistic for C2ABS2-GLPK score

338 either in the development cohort (c-statistic, $0.811 ; 95 \%$ CI, 0.786-0.836) or the external

339 validation cohort (c-statistic, $0.787 ; 95 \%$ CI, 0.756-0.818) were all significantly higher than 
340 those of the GRACE score (c-statistic, 0.728; 95\% CI, 0.697-0.759) $(p<0.001)$, KAMIR score (c-

341 statistic, 0.783; 95\% CI, 0.758-0.808) $(p<0.001)$ and CAMI score (c-statistic, 0.558; 95\% CI,

$3420.523-0.593)(p<0.001)$, which reflects the higher prognostic value of the C2ABS2-GLPK score

343 for the prediction of 5-year mortality in Chinese patients with AMI (Table S8).

344 To further assess the clinical utility of the C2ABS2-GLPK score, we also performed decision

345 curve analyses to compare this score with the GRACE score, KAMIR score and CAMI score.

346 The results showed that the C2ABS2-GLPK score had better clinical utility across the threshold

347 of 5-year all-cause mortality applicable to more than $50 \%$ of both the development cohort and

348 the external validation cohort (Fig. 4).

349

350 Sensitivity Analysis

351 To further confirm the reliability of the C2ABS2-GLPK model, we performed sensitivity

352 analyses by using complete case data. The results showed that the discrimination with the C-

353 statistic based on complete case data was 0.805 (95\% CI, 0.774-0.836) in the development cohort

354 and $0.780(95 \% \mathrm{CI}, 0.743-0.817)$ in the external validation cohort, which was similar to that with

355 C-statistics of 0.811 (95\% CI, 0.786-0.836) and 0.787 (95\% CI, 0.756-0.818) in the imputed

356 development and validation datasets, respectively (Table S11). 
358 Subgroup Validation

359 Consistent with the performance in the whole cohort, the predictive performance of the

360 C2ABS2-GLPK score was robust across all subgroups, with C-statistics ranging from 0.770 to

3610.832 in subgroups of patients according to sex, history of smoking, types of AMI, and

362 comorbidities of diabetes, obesity and hypercholesterolaemia in the internal and external

363 validation cohorts. Furthermore, the performance of the C2ABS2-GLPK score was superior to

364 that of the GRACE score (c-statistics range: 0.684 to 0.765 ), the KAMIR score (c-statistics range:

3650.744 to 0.807 ), and the CAMI score (c-statistics range: 0.477 to 0.580 ) in the indicated

366 subgroups (Table S12, Fig. S3).

Discussion

369 In this study, we developed and validated a clinical prognostic score (C2ABS2-GLPK score)

370 assessed within $24 \mathrm{~h}$ of admission to predict 5-year mortality among Chinese patients with AMI

371 in two independent cohorts, which allows for the potential collinearity of independent predictors

372 and overfittings (Tibshirani, 1997; McClelland et al., 2015). Overall, the C2ABS2-GLPK score

373 had satisfactory discrimination and calibration performance for the 5-year prediction of all-cause

374 mortality in Chinese patients with AMI. With reference to the GRACE scoring system, the

375 patients in the medium-risk group with a C2ABS2-GLPK score between 149 and 218 might be 
376 suggested to have guideline-indicated pharmacotherapies and evidence-based care. Patients with

377 a C2ABS2-GLPK score above 219 in the high-risk group of C2ABS2-GLPK score might be

378 recommended to have 'up to standard' guideline-indicated care, intensified therapy, and

379 additional lifestyle modifications and follow-up to improve their long-term survival (Hall M et

380 al.,2018). The performance and risk stratification abilities of the C2ABS2-GLPK score warrant

381 further validation in prospective studies.

382 Over the past 30 years, some risk scores for predicting long-term mortality in patients with

383 AMI, such as the GRACE (Kozieradzka et al., 2011), KAMIR (Kim et al., 2011), SAMI (Plakht

384 et al., 2006), PAMI (Addala et al., 2004), TIMI (Kozieradzka et al., 2011) and Zwolle scores

385 (Kozieradzka et al., 2011), have been developed and validated. However, these scores were

386 based on Western and Korean populations due to differences in medical interventions, responses

387 to therapy, economic levels and genetic backgrounds across countries; consequently, they may

388 not be well suited to predict long-term mortality for Chinese AMI patients. Recently, the China

389 AMI (CAMI) registry, a nationwide registry of hospitalized patients with AMI, was designed

390 and launched in China. Although there are plans for predicting 2-year mortality of AMI in the

391 CAMI database, the CAMI score is currently focused only on in-hospital mortality (Xu et al.,

392 2016; Fu et al., 2018; Tang et al., 2019). Additionally, the CAMI score might not be easily

393 accessible in the clinic due to its two scoring systems specific to CAMI-STEMI and CAMI-

394 NSTEMI. Therefore, we developed the C2ABS2-GLPK score for predicting long-term mortality 
395 in Chinese AMI patients to be simple and easily available in clinical practice. We also validated

396 that the C2ABS2-GLPK score outperformed the GRACE, KAMIR and CAMI scores in

397 estimating the 5-year risk of death in AMI patients. In addition, the C2ABS2-GLPK score, which

398 was established using clinical parameters and medical intervention at admission, may guide the

399 optimization of interventional strategies under emergency circumstances.

400 Compared to the widely used classic GRACE scoring system, our C2ABS2-GLPK score

401 might be somewhat superior in the following aspects. First, it is well known that the GRACE

402 score for predicting more than 6-month mortality in AMI patients is assessed at hospital

403 discharge (Eagle KA et al., 2004). However, the C2ABS2-GLPK score is calculated within $24 \mathrm{~h}$

404 of admission and might be more timely for optimizing the therapeutic and monitoring regimen

405 during hospitalization to eventually improve long-term survival for AMI patients in China.

406 Second, the GRACE risk model was developed and validated based on data from 1999 to 2003

407 (Allen LA et al., 2007). With advances in the treatment of AMI, the GRACE score may not be

408 suitable for current AMI patients. Our C2ABS2-GLPK score integrated advanced interventional

409 procedures and medication to fill in the gaps of the current scores in predicting long-term

410 mortality for AMI patients. Third, compared with the GRACE score, the C2ABS2-GLPK score

411 in the present study increased the accuracy of predicting long-term prognosis by adding a new

412 dimension, the status of target organs such as the heart and brain, which has been proven to

413 predict long-term mortality in patients with AMI (Israr MZ et al., 2018; Richards AM et al., 
414 2003; Ndrepepa G et al., 2006; Taylor HA et al., 2007; Brammås A et al., 2013). Fourth, in

415 comparison to the GRACE score, the C2ABS2-GLPK score places more emphasis on the

416 essential role of statins in the early treatment of AMI, which has recently been considered to be

417 an independent predictor of one-year major adverse cardiovascular events in patients with AMI

418 (Kim MC et al., 2019).

419 The C2ABS2-GLPK score provided a comprehensive evaluation of cardiac function from

420 clinical manifestation (Killip classification), serum biochemical indicators (NT-proBNP), and

421 structure of the cardiac chamber(cardiac ultrasound) in the acute phase. The Killip classification

422 primarily considers cardiac findings during physical examination, which has proven to be

423 essential in predicting mortality in AMI patients in dozens of studies (Khot et al., 2003; Stebbins

424 et al., 2010; Fu et al., 2018; Song et al., 2018; Chen, Han \&Luo, 2019; Meyer et al., 2019;

425 Wang et al., 2021). However, this classification may not be sensitive to detecting asymptomatic

426 heart failure. NT-proBNP, a sensitive biomarker for heart failure, is secreted from the atria and

427 ventricles and is increased in both symptomatic and asymptomatic patients with cardiac

428 dysfunction. Furthermore, cardiac ultrasound adds a definitive diagnosis and evaluation of the

429 structure and function of each cardiac chamber, valve, and attachments. Therefore, integration of

430 the three indicators improved both the sensitivity and specificity of the evaluation of cardiac

431 function. 
432 The PLATelet inhibition and patient Outcomes (PLATO) trial reported that ACS patients with a

433 history of stroke or transient ischaemic attack (TIA) had higher rates of 1 -year death $(10.5 \%)$

434 than those without a history of stroke or TIA (4.9\%) (Mahaffey et al., 2014). A systematic review

435 showed that AMI patients with a history of stroke had a higher risk of mortality than patients

436 without stroke at the one-year follow-up (Johansson et al., 2017). Our study also identified that

437 prior stroke or acute stroke at admission is an important predictor of 5-year mortality in patients

438 with AMI.

439 Numerous studies have shown that statins benefit in-hospital and long-term survival in AMI

440 patients via multiple mechanisms, such as lipid reduction, anti-inflammation and plaque

441 stabilization (Heart Protection Study Collaborative Group, 2002; Merx et al., 2005; Sim et al.,

442 2013). Existing evidence from a Chilean registry showed that early initiation of statins improved

443 in-hospital survival in patients with AMI (Martínez et al., 2013). Concordant with these findings,

444 we study revealed that early statin therapy improved long-term survival in patients with AMI.

445 Cardiac arrest has been well demonstrated to substantially increase in-hospital and 30-day

446 mortality risk (Newby et al., 1998;Fordyce et al., 2016). However, studies have shown that AMI

447 patients with perihospital cardiac arrest did not have higher 1-year mortality than those without

448 prehospitalization cardiac arrest (Lee et al., 2014;Fordyce et al., 2016). Our study revealed that

449 cardiac arrest is the strongest predictor for long-term mortality in AMI patients. 
450 There are some limitations of our study. First, the modest sample size used to construct the

451 risk score and validation may influence the accuracy of the model. Future studies with a larger

452 population are warranted. Second, the cohorts for score development and validation are from one

453 province in China, which could limit the generalizability of the score to patients in other areas of

454 China. Additional validation studies in patients with AMI from areas outside Hunan Province is

455 worth performing in further studies. Third, this model was based on the retrospective

456 development and validation cohorts, and prospective studies may further increase the reliability

457 of this model. Fourth, 45 candidate variables were selected and used to predict 5-year mortality.

458 There is a potential risk of overfitting during score development; Fifth, a retrospective design

459 cannot rule out the influence of unmeasured confounders, such as economic status and

460 compliance, that may impact long-term mortality. Sixth, since we selected the candidate

461 variables based on a literature review, other potential factors impacting long-term mortality in

462 AMI patients were possibly excluded during the initial variable selection. Inevitable selection

463 bias may exist and lead to the overestimation of risk. Future studies are warranted to confirm the

464 robustness of our score.

\section{Conclusions}

467 In summary, the C2ABS2-GLPK score was established to estimate 5-year mortality in AMI

468 patients in China based on ten variables that are commonly measured at hospital admission. The 
469 C2ABS2-GLPK score showed good performance in predicting long-term outcomes in AMI

470 patients, however, warrant to be further validation in additional cohorts and in prospective

471 settings is warranted. This tool may help physicians identify patients who are at high risk of

472 long-term mortality, thus optimizing medical treatments and eventually improving long-term

473 survival of AMI patients in China.

474

475 Acknowledgments

476 None.

477

478 ADDITIONAL INFORMATION AND DECLARATIONS

479

480 Grant Disclosures

481 The following grant information was disclosed by the authors:

482 Key Research and Development Plan of Hunan Province: S2021GCZDYF1348.

483 National Natural Science Foundation of China: 81870171, 81800393, 81770403 and 81974054. 
484 National Key Research and Development Projects: 2019YFF0216300， 2019YFF0216305 and 4852018 YFC1311300.

\section{Author Contributions}

488 Yan Tang and Jingjing Cai conceived and designed the experiments, analyzed the data, prepared

489 figures and/or tables, revised it critically for important content, authored or reviewed drafts of the 490 paper, and approved the final draft.

491 Yan Tang, Yuanyuan Bai and Yuanyuan Chen performed the experiments, analyzed the data, 492 revised it critically for important content, and authored or reviewed drafts of the paper.

493 Yan Tang and Yuanyuan Bai prepared figures and/or tables, and approved the final draft.

494 Xuejing Sun, Yunmin Shi, Tian He, Mengqing Jiang, Yujie Wang, Mingxing Wu, Zhiliu Peng 495 and Suzhen Liu analyzed the data, revised it critically for important content, and authored or 496 reviewed drafts of the paper.

497 Weihong Jiang, Yao Lu, and Hong Yuan analyzed the data, revised it critically for important 498 content, and authored or reviewed drafts of the paper. 
500 Human Ethics

501 The following information was supplied relating to ethical approvals (i.e., approving body

502 and any reference numbers):

503 The Development database was approved by the institutional review boards (IRB) of the Third

504 Xiangya Hospital of Central South University [(2019) Ethical Approval No. S489]. The

505 validation database was approved by the Xiangtan Central Hospital granted Ethical approval

506 [(2020) Ethical Approval No. 11-001]. Since this study is a retrospective study, it only collects

507 the clinical data of patients with no treatment plans and will not bring risks to the patient's

508 physiology. Therefore, the need for informed consent was waived in our study.

509

510 Supplemental Information

511 Supplemental information for this article can be found online.

512

\section{Supplementary Materials}

514 Figure S1-S3

515 Table S1-S12 
517 References

518 Addala S, Grines CL, Dixon SR, Stone GW, Boura JA, Ochoa AB, Pellizzon G, O'Neill

519 WW, Kahn JK. 2004. Predicting mortality in patients with ST-elevation myocardial infarction

520 treated with primary percutaneous coronary intervention (PAMI risk score). Am J Cardiol

521 93:629-32. DOI 10.1016/j.amjcard.2003.11.036.

522 Allen LA, Tang EW, Wong CK, Herbison P. 2007. Global Registry of Acute Coronary Events

523 (GRACE) hospital discharge risk score accurately predicts long-term mortality post acute

524 coronary syndrome. Am Heart J 153:29-35. DOI 10.1016/j.ahj.2006.10.004.

525 Azur MJ, Stuart EA, Frangakis C, Leaf PJ. 2011. Multiple imputation by chained equations:

526 what is it and how does it work? Int J Methods Psychiatr Res 20:40-9. DOI 10.1002/mpr.329.

527 Brammås A, Jakobsson S, Ulvenstam A, Mooe T. 2013. Mortality after ischemic stroke in

528 patients with acute myocardial infarction: predictors and trends over time in Sweden. Stroke

529 44:3050-5. DOI 10.1161/STROKEAHA.113.001434.

530 Camp RL, Dolled-Filhart M, Rimm DL. 2004. X-tile: a new bio-informatics tool for

531 biomarker assessment and outcome-based cut-point optimization. Clin Cancer Res 10:7252-9.

532 DOI 10.1158/1078-0432.CCR-04-0713. 
533 Chang J, Liu X, Sun Y. 2017. Mortality due to acute myocardial infarction in China from 1987

534 to 2014: secular trends and age-period-cohort effects. Int J Cardiol 227:229-238. DOI

535 10.1016/j.ijcard.2016.11.130.

536 Chen L, Han L, Luo J. 2019. Risk Factors for Predicting Mortality among Old Patients with 537 Acute Myocardial Infarction during Hospitalization. Heart Surg Forum 22:E165-E169. DOI $538 \quad 10.1532 / \mathrm{hsf} .2265$.

D. S. Sim, M.H. Jeong, K.H. Cho,Y.K. Ahn,Y.J. Kim, S.C. Chae, T.J. Hong, Chae, I.W.

Seong, J.K. Chae,C.J. Kim, M.C. Cho, S.W. Rha, J.H. Bae, K.B. Seung, S.J. Park. 2013.

541 Effect of early statin treatment in patients with cardiogenic shock complicating acute myocardial

542 infarction. Korean Circ J 43: 100-9. DOI 10.4070/kcj.2013.43.2.100.

543 Eagle KA, Lim MJ, Dabbous OH, Pieper KS, Goldberg RJ, Van de Werf F, Goodman SG,

544 Granger CB, Steg PG, Gore JM, Budaj A, Avezum A, Flather MD, Fox KA, GRACE

545 Investigators. 2004. A validated prediction model for all forms of acute coronary syndrome:

546 estimating the risk of 6-month postdischarge death in an international registry. JAMA 291:2727-

547 33. DOI 10.1001/jama.291.22.2727.

548 Fordyce CB, Wang TY, Chen AY, Thomas L, Granger CB, Scirica BM, Henry TD, Wong

549 GC, Ramanathan K, Hansen CM, Kragholm K, Peterson ED, Anderson ML. 2016. Long- 
550 Term Post-Discharge Risks in Older Survivors of Myocardial Infarction With and Without Out-

551 of-Hospital Cardiac Arrest. J Am Coll Cardiol 67:1981-90. DOI 10.1016/j.jacc.2016.02.044.

552 Fox KA, Carruthers KF, Dunbar DR, Graham C, Manning JR, De Raedt H, Buysschaert I,

553 Lambrechts D, Van de Werf F. 2010. Underestimated and under-recognized: the late

554 consequences of acute coronary syndrome (GRACE UK-Belgian Study). Eur Heart J 31:2755-

555 64. DOI 10.1093/eurheartj/ehq326.

556 Fu R, Song C, Yang J, Wang Y, Li B, Xu H, Gao X, Li W, Liu J, Dou K, Yang Y, From

557 CAMI Registry Study Group. 2018. CAMI-NSTEMI Score-China Acute Myocardial

558 Infarction Registry-Derived Novel Tool to Predict In-Hospital Death in Non-ST Segment

559 Elevation Myocardial Infarction Patients. Circ J 82:1884-1891. DOI 10.1253/circj.CJ-17-1078.

Hall M, Bebb OJ, Dondo TB, Yan AT, Goodman SG, Bueno H, Chew DP, Brieger D, Batin

PD, Farkouh ME, Hemingway H, Timmis A, Fox KAA, Gale CP. 2018. Guideline-indicated treatments and diagnostics, GRACE risk score, and survival for non-ST elevation myocardial infarction. Eur Heart J 42:3798-3806. DOI 10.1093/eurheartj/ehy517.

564 Harrell FE, Lee KL, Mark DB. 1996. Multivariable prognostic models: issues in developing

565 models, evaluating assumptions and adequacy, and measuring and reducing errors.Stat Med

566 15:361-87. DOI 10.1002/(SICI)1097-0258(19960229)15:4<361::AID-SIM168>3.0.CO;2-4.

567 Heart Protection Study Collaborative Group. 2002. MRC/BHF Heart Protection Study of 
568 cholesterol lowering with simvastatin in 20,536 high-risk individuals: a randomised placebo-

569 controlled trial. Lancet 360:7-22. DOI 10.1016/S0140-6736(02)09327-3.

570 Henderson RA, Jarvis C, Clayton T, Pocock SJ, Fox KA. 2015. 10-Year Mortality Outcome

571 of a Routine Invasive Strategy Versus a Selective Invasive Strategy in Non-ST-Segment

572 Elevation Acute Coronary Syndrome: The British Heart Foundation RITA-3 Randomized Trial.

$573 J$ J Am Coll Cardiol 5:511-20. DOI 10.1016/j.jacc.2015.05.051.

574 Hippisley-Cox J, Coupland C, Brindle P. 2013. Derivation and validation of QStroke score for

575 predicting risk of ischaemic stroke in primary care and comparison with other risk scores: a

576 prospective open cohort study. BMJ 346:f2573. DOI 10.1136/bmj.f2573.

577 Hyder O, Marques H, Pulitano C, Marsh JW, Alexandrescu S, Bauer TW, Gamblin TC,

578 Sotiropoulos GC, Paul A, Barroso E, Clary BM, Aldrighetti L, Ferrone CR, Zhu AX,

579 Popescu I, Gigot JF, Mentha G, Feng S, Pawlik TM. 2014. A nomogram to predict long-term

580 survival after resection for intrahepatic cholangiocarcinoma: an Eastern and Western experience.

581 JAMA Surg 149:432-8. DOI 10.1001/jamasurg.2013.5168.

582 Israr MZ, Heaney LM, Ng LL, Suzuki T. 2018. B-type natriuretic peptide molecular forms for

583 risk stratification and prediction of outcome after acute myocardial infarction. Am Heart $J$

584 200:37-43. DOI 10.1016/j.ahj.2018.02.016. 
585 Johansson S, Rosengren A, Young K, Jennings E. 2017. Mortality and morbidity trends after

586 the first year in survivors of acute myocardial infarction: a systematic review. BMC Cardiovasc

587 Disord 17:53. DOI 10.1186/s12872-017-0482-9.

588 Kalbfleisch JD, Prentice RL. 2002. The Statistical Analysis of Failure Time Data. second

589 edition. New York: Wiley.

590 Kalderstam J, Edén P, Bendahl PO, Strand C, Fernö M, Ohlsson M. 2013. Training artificial

591 neural networks directly on the concordance index for censored data using genetic algorithms.

592 Artif Intell Med 58:125-32.DOI 10.1016/j.artmed.2013.03.001.

593 Khan NA, Grubisic M, Hemmelgarn B, Humphries K, King KM, Quan H. 2010. Outcomes

594 after acute myocardial infarction in South Asian, Chinese, and white patients. Circulation

122:1570-7. DOI 10.1161/CIRCULATIONAHA.109.850297.

Khot UN, Jia G, Moliterno DJ, Lincoff AM, Khot MB, Harrington RA, Topol EJ. 2003.

597 Prognostic importance of physical examination for heart failure in non-ST-elevation acute

598 coronary syndromes: the enduring value of Killip classification. JAMA 290:2174-81. DOI

599 10.1001/jama.290.16.2174.

600 Kim HK, Jeong MH, Ahn Y, Kim JH, Chae SC, Kim YJ, Hur SH, Seong IW, Hong TJ,

601 Choi DH, Cho MC, Kim CJ, Seung KB, Chung WS, Jang YS, Rha SW, Bae JH, Cho JG,

602 Park SJ, Other Korea Acute Myocardial Infarction Registry Investigators. 2011. Hospital 
603 discharge risk score system for the assessment of clinical outcomes in patients with acute 604 myocardial infarction (Korea Acute Myocardial Infarction Registry [KAMIR] score). Am J 605 Cardiol 107:965-971.e1. DOI 10.1016/j.amjcard.2010.11.018.

606 Kim MC, Ahn YK, Cho JY, Lee KH, Sim DS, Yoon NS, Yoon HJ, Kim KH, Hong YJ, Park

607 HW, Kim JH, Jeong MH, Cho JG, Park JC, Chang KY, Seung KB. 2019. Benefit of Early

608 Statin Initiation within 48 Hours after Admission in Statin-Naïve Patients with Acute Myocardial

609 Infarction Undergoing Percutaneous Coronary Intervention. Korean Circ J 49: 419-433. DOI

$610 \quad 10.4070 / \mathrm{kcj} .2018 .0341$.

611 Knight SR, Ho A, Pius R, Buchan I, Carson G, Drake TM, Dunning J, Fairfield CJ,

612 Gamble C, Green CA, Gupta R, Halpin S, Hardwick HE, Holden KA, Horby PW, Jackson

613 C, Mclean KA, Merson L, Nguyen-Van-Tam JS, Norman L, Noursadeghi M, Olliaro PL,

614 Pritchard MG, Russell CD, Shaw CA, Sheikh A, Solomon T, Sudlow C, Swann OV, Turtle

615 LC, Openshaw PJ, Baillie JK, Semple MG, Docherty AB, Harrison EM, ISARIC4C

616 investigators. 2020. Risk stratification of patients admitted to hospital with covid-19 using the

617 ISARIC WHO Clinical Characterisation Protocol: development and validation of the 4C

618 Mortality Score. BMJ 370:m3339. DOI 10.1136/bmj.m3339.

619 Kozieradzka A, Kamiński KA, Maciorkowska D, Olszewska M, Dobrzycki S, Nowak K,

620 Kralisz P, Prokopczuk P, Musial WJ. 2011. GRACE, TIMI, Zwolle and CADILLAC risk 
621 scores--do they predict 5-year outcomes after ST-elevation myocardial infarction treated

622 invasively? Int J Cardiol 148:70-5. DOI 10.1016/j.ijcard.2009.10.026.

623 Kruppa J, Liu Y, Biau G, Kohler M, König IR, Malley JD, Ziegler A. 2014. Probability

624 estimation with machine learning methods for dichotomous and multicategory outcome: theory.

625 Biom J 4:534-63. DOI 10.1002/bimj.201300068.

Laribi S, Aouba A, Resche-Rigon M, Johansen H, Eb M, Peacock FW, Masip J, Ezekowitz

JA, Cohen-Solal A, Jougla E, Plaisance P, Mebazaa A. 2014. Trends in death attributed to myocardial infarction, heart failure and pulmonary embolism in Europe and Canada over the last decade. $Q J M$ 107:813-20. DOI 10.1093/qjmed/hcu083.

Lee KH, Jeong MH, YoungkeunAhn, Kim SS, Rhew SH, Jeong YW, Jang SY, Cho JY,

Infarction Registry) Investigators. 2014. One-year clinical impact of cardiac arrest in patients

DOI 10.1016/j.ijcard.2014.05.002.

637 From China PEACE Collaborative Group. 2015. ST-segment elevation myocardial infarction

638 in China from 2001 to 2011 (the China PEACE-Retrospective Acute Myocardial Infarction 
639 Study): a retrospective analysis of hospital data. Lancet 385:441-51. DOI 10.1016/S0140-

$640 \quad 6736(14) 60921-1$.

641 Mahaffey KW, Held C, Wojdyla DM, James SK, Katus HA, Husted S, Steg PG, Cannon

642 CP, Becker RC, Storey RF, Khurmi NS, Nicolau JC, Yu CM, Ardissino D, Budaj A,

643 Morais J, Montgomery D, Himmelmann A, Harrington RA, Wallentin L, PLATO

644 Investigators. 2014. Ticagrelor effects on myocardial infarction and the impact of event

645 adjudication in the PLATO (Platelet Inhibition and Patient Outcomes) trial. $J$ Am Coll Cardiol

646 63:1493-9. DOI 10.1016/j.jacc.2014.01.038.

647 Martínez G, Rigotti A, Acevedo M, Navarrete C, Rosales J, Giugliano RP, Corbalán R.

648 2013. Cholesterol levels and the association of statins with in-hospital mortality of myocardial

649 infarction patients insights from a Chilean registry of myocardial infarction. Clin Cardiol

650 36:305-11. DOI 10.1002/clc.22110.

651 McClelland RL, Jorgensen NW, Budoff M, Blaha MJ, Post WS, Kronmal RA, Bild DE,

652 Shea S, Liu K, Watson KE, Folsom AR, Khera A, Ayers C, Mahabadi AA, Lehmann N,

653 Jöckel KH, Moebus S, Carr JJ, Erbel R, Burke GL. 2015. 10-Year Coronary Heart Disease

654 Risk Prediction Using Coronary Artery Calcium and Traditional Risk Factors: Derivation in the

655 MESA (Multi-Ethnic Study of Atherosclerosis) With Validation in the HNR (Heinz Nixdorf 
656 Recall) Study and the DHS (Dallas Heart Study). J Am Coll Cardiol 66:1643-53. DOI

657 10.1016/j.jacc.2015.08.035.

658 Merx MW, Liehn EA, Graf J, van de Sandt A, Schaltenbrand M, Schrader J, Hanrath P,

659 Weber C. 2005. Statin treatment after onset of sepsis in a murine model improves survival.

660 Circulation 112: 117-24. DOI 10.1161/CIRCULATIONAHA.104.502195.

661 Meyer MR, Bernheim AM, Kurz DJ, O'Sullivan CJ, Tüller D, Zbinden R, Rosemann T,

662 Eberli FR. 2019. Gender differences in patient and system delay for primary percutaneous

663 coronary intervention: current trends in a Swiss ST-segment elevation myocardial infarction

664 population. Eur Heart J Acute Cardiovasc Care 8:283-290. DOI 10.1177/2048872618810410.

665 Moons KG, Altman DG, Reitsma JB, Ioannidis JP, Macaskill P, Steyerberg EW, Vickers

666 AJ, Ransohoff DF, Collins GS. 2015. Transparent Reporting of a multivariable prediction

667 model for Individual Prognosis or Diagnosis (TRIPOD): explanation and elaboration. Ann Intern

668 Med 162: W1-73. DOI 10.7326/M14-0698.

669 Ndrepepa G, Braun S, Mehilli J, von Beckerath N, Nekolla S, Vogt W, Schwaiger M,

670 Schömig A, Kastrati A. 2006. N-terminal pro-brain natriuretic peptide on admission in patients

671 with acute myocardial infarction and correlation with scintigraphic infarct size, efficacy of

672 reperfusion, and prognosis. Am J Cardiol 97:1151-6. DOI 10.1016/j.amjcard.2005.11.029. 
673 Newby KH, Thompson T, Stebbins A, Topol EJ, Califf RM, Natale A. 1998. Sustained

674 ventricular arrhythmias in patients receiving thrombolytic therapy: incidence and outcomes. The

675 GUSTO Investigators. Circulation 98:2567-73. DOI 10.1161/01.cir.98.23.2567.

676 Plakht Y, Shiyovich A, Weitzman S, Fraser D, Zahger D, Gilutz H. 2012. A new risk score

677 predicting 1- and 3-year mortality following acute myocardial infarction Soroka Acute

678 Myocardial Infarction (SAMI) Project. Int J Cardiol 154:173-9. DOI

679 10.1016/j.ijcard.2010.09.014.

680 Reed GW, Rossi JE, Cannon CP. 2017. Acute myocardial infarction. Lancet 389:197-210.

681 DOI 10.1016/S0140-6736(16)30677-8.

682 Richards AM, Nicholls MG, Espiner EA, Lainchbury JG, Troughton RW, Elliott J,

683 Frampton C, Turner J, Crozier IG, Yandle TG. 2003. B-type natriuretic peptides and ejection

684 fraction for prognosis after myocardial infarction. Circulation 107:2786-92. DOI

685 10.1161/01.CIR.0000070953.76250.B9.

686

687

688

689

690
Rogers WJ, Frederick PD, Stoehr E, Canto JG, Ornato JP, Gibson CM, Pollack CV, Gore

JM, Chandra-Strobos N, Peterson ED, French WJ. 2008. Trends in presenting characteristics and hospital mortality among patients with ST elevation and non-ST elevation myocardial infarction in the National Registry of Myocardial Infarction from 1990 to 2006. Am Heart J 156:1026-34. DOI 10.1016/j.ahj.2008.07.030. 
691 Roth GA, Mensah GA, Johnson CO, Addolorato G, Ammirati E, Baddour LM, Barengo

692 NC, Beaton AZ, Benjamin EJ, Benziger CP, Bonny A, Brauer M, Brodmann M, Cahill TJ,

693 Carapetis J, Catapano AL, Chugh SS, Cooper LT, Coresh J, Criqui M, DeCleene N, Eagle

694 KA, Emmons-Bell S, Feigin VL, Fernández-Solà J, Fowkes G, Gakidou E, Grundy SM, He

695 FJ, Howard G, Hu F, Inker L, Karthikeyan G, Kassebaum N, Koroshetz W, Lavie C,

696 Lloyd-Jones D, Lu HS, Mirijello A, Temesgen AM, Mokdad A, Moran AE, Muntner P,

697 Narula J, Neal B, Ntsekhe M, Moraes de Oliveira G, Otto C, Owolabi M, Pratt M,

698 Rajagopalan S, Reitsma M, Ribeiro ALP, Rigotti N, Rodgers A, Sable C, Shakil S, Sliwa-

699 Hahnle K, Stark B, Sundström J, Timpel P, Tleyjeh IM, Valgimigli M, Vos T, Whelton PK,

700 Yacoub M, Zuhlke L, Murray C, Fuster V, From GBD-NHLBI-JACC Global Burden of

701 Cardiovascular Diseases Writing Group. 2020. Global Burden of Cardiovascular Diseases

702 Writing Group. Global Burden of Cardiovascular Diseases and Risk Factors, 1990-2019: Update

703 From the GBD 2019 Study. J Am Coll Cardiol. DOI 10.1016/j.jacc.2020.11.010.

704 Royston P, Altman DG. 2013. External validation of a Cox prognostic model: principles and

705 methods. BMC Med Res Methodol 13: 33. DOI 10.1186/1471-2288-13-33.

706 Song C, Fu R, Dou K, Yang J, Xu H, Gao X, Li W, Gao G, Zhao Z, Liu J, Yang Y. 2018.

707 The CAMI-score: A Novel Tool derived From CAMI Registry to Predict In-hospital Death

708 among Acute Myocardial Infarction Patients. Sci Rep 8:9082. DOI 10.1038/s41598-018-26861-z. 
709 Song J, Murugiah K, Hu S, Gao Y, Li X, Krumholz HM, Zheng X, From China PEACE

710 Collabortive Group. 2020. Incidence, predictors, and prognostic impact of recurrent acute

711 myocardial infarction in China. Heart. DOI 10.1136/heartjnl-2020-317165.

712 Speiser JL, Durkalski VL, Lee WM. 2015. Random forest classification of etiologies for an

713 orphan disease. Stat Med 5:887-99. DOI 10.1002/sim.6351.

714 Stebbins A, Mehta RH, Armstrong PW, Lee KL, Hamm C, Van de Werf F, James S,

715 Toftegaard-Nielsen T, Seabra-Gomes R, White HD, Granger CB.2010. Assessment of

716 Pexelizumab in Acute Myocardial Infarction (APEX AMI Investigators). A model for predicting

717 mortality in acute ST-segment elevation myocardial infarction treated with primary percutaneous

718 coronary intervention: results from the Assessment of Pexelizumab in Acute Myocardial

719 Infarction Trial. Circ Cardiovasc Interv 3:414-22. DOI

720

10.1161/CIRCINTERVENTIONS.109.925180.

721 Steyerberg EW. 2008. Case study on survival analysis: prediction of secondary cardiovascular

722 events. Clinical Prediction Models: A Practical Approach to Development, Validation, and

723 Updating. New York: Springer; 436-437.

724 Steyerberg EW, Vickers AJ, Cook NR, Gerds T, Gonen M, Obuchowski N, Pencina MJ,

725 Kattan MW. 2010. Assessing the performance of prediction models: a framework for traditional

726 and novel measures. Epidemiology 21:128-38. DOI 10.1097/EDE.0b013e3181c30fb2. 
727 Szummer K, Jernberg T, Wallentin L. 2019. From Early Pharmacology to Recent

728 Pharmacology Interventions in Acute Coronary Syndromes: JACC State-of-the-Art Review. $J$

729 Am Coll Cardiol 74:1618-1636. DOI 10.1016/j.jacc.2019.03.531.

730 Tang C, Luo E, Wang D, Yan G, Qiao Y, Zhu B, Liu B, Hou J. 2019. Usefulness of

731 Haemoglobin Level Combined with CAMI-STEMI Score for Predicting MACCE in Patients

732 with Acute ST-Elevation Myocardial Infarction after PCI. Biomed Res Int 2019:8534752. DOI

$73310.1155 / 2019 / 8534752$.

734 Taylor HA, Penman AD, Han H, Dele-Michael A, Skelton TN, Fox ER, Benjamin EJ,

735 Arnett DK, Mosley TH. 2007. Left ventricular architecture and survival in African-Americans

736 free of coronary heart disease (from the Atherosclerosis Risk in Communities [ARIC] study). $\mathrm{Am}$

737 J Cardiol 99:1413-20. DOI 10.1016/j.amjcard.2006.12.065.

738 Tibshirani R.1997. The lasso method for variable selection in the Cox model. Stat Med 16:385-

739 95.DOI $10.1002 /($ sici)1097-0258(19970228)16:4<385::aid-sim380>3.0.co;2-3.

740 Vickers AJ, Elkin EB.2006. Decision curve analysis: a novel method for evaluating prediction

741 models. Med Decis Making 26:565-74. DOI 10.1177/0272989X06295361.

742 Waljee AK, Mukherjee A, Singal AG, Zhang Y, Warren J, Balis U, Marrero J, Zhu J,

743 Higgins PD. 2013. Comparison of imputation methods for missing laboratory data in medicine.

744 BMJ Open 3. DOI 10.1136/bmjopen-2013-002847. 
745 Wang SJ, Cheng ZX, Fan XT, Lian YG.2021. Development of an optimized risk score to

746 predict short-term death among acute myocardial infarction patients in rural China. Clin Cardiol.

747 DOI 10.1002/clc.23598.

748 Xu H, Li W, Yang J, Wiviott SD, Sabatine MS, Peterson ED, Xian Y, Roe MT, Zhao W,

749 Wang Y, Tang X, Jia X, Wu Y, Gao R, Yang Y, From CAMI Registry study group. 2016.

750 The China Acute Myocardial Infarction (CAMI) Registry: A national long-term registry-

751 research-education integrated platform for exploring acute myocardial infarction in China. $\mathrm{Am}$

752 Heart J 175:193-201.e3. DOI 10.1016/j.ahj.2015.04.014.

753 Yeh RW, Sidney S, Chandra M, Sorel M, Selby JV, Go AS. 2010. Population trends in the

754 incidence and outcomes of acute myocardial infarction. $N$ Engl J Med 362:2155-65.DOI

755 10.1056/NEJMoa0908610.

Zdanyte M, Wrazidlo RW, Kaltenbach S, Groga-Bada P, Gawaz M, Geisler T, Rath D.

757

2020. Predicting 1-, 3- and 5-year outcomes in patients with coronary artery disease: A 
Figure Legends

761 Figure 1 Flowchart for Patient Selection and Distribution of the Development and

762 Validation Cohorts.

763 Abbreviations:AMI:acute myocardial infarction.

Figure 2 Nomogram for C2ABS2-GLPK Score to Predict 1-, 2-, 3-, 4-, 5-Year All-Cause

Mortality in Patients with AMI and Kaplan-Meier Survival Curves Stratified by C2ABS2-

GLPK Score.

(A) Nomogram for Predicting 1-, 2-, 3-, 4-, 5-Year All-Cause Mortality among Patients with

AMI. (B) Kaplan-Meier Survival Curves for AMI Patients with Risk Stratified by C2ABS2-

Survival Curves for AMI Patients with Risk Stratified by C2ABS2-GLPK Score according to the

X-tile Analysis in the External Validation Cohort.

773 Notes: Nomogram for the prediction of 1-, 2-, 3-, 4-, 5-Year All-cause Mortality-Free in Patients with AMI. The patient's age is located on the row labeled “Age, y", the patient's left ventricular end-diastolic diameter is located on the row labeled "Left Ventricular end-diastolic Diameter, mm", the patient's fasting blood glucose level is located on the row labeled "Fasting Blood

777 Glucose, $\mathrm{mmol} / \mathrm{L}$ ", the patient's creatinine level is located on the row labeled "Creatinine, 
$778 \mu \mathrm{mol} / \mathrm{L}$ ", the patient's NT-proBNP level is located on the row labeled "NT-proBNP, pg/ml" ,

779 the patient's Killip classification is located on the row labeled "Killip, classifications" , and a

780 straight line is drawn up to the row labeled "Points" to determine the corresponding points. This

781 process is repeated for each of the remaining factors by drawing a straight line to the "Points"

782 row to determine the points associated with each factor. After summing the total points, one

783 locates the appropriate total point number and draws a straight line from this to the rows labeled

784 'Predicted 1-, 2-, 3-, 4-, 5-Year All-cause Mortality-Free probability to determine the patient's

785 predicted survival probability.

786 Abbreviations: AMI: acute myocardial infarction; NT-proBNP: N-terminal pro-brain natriuretic

787 peptide; PCI: percutaneous transluminal coronary intervention.

Figure 3 Calibration of C2ABS2-GLPK Score in the Development Cohort and Validation

790 Cohort.

791 (A) Agreement between the Deciles of Observed vs Predicted 5-Year Mortality in the Cox

792 Model of the Development Cohort. (B) Agreement between the Deciles of Observed vs Predicted

793 5-Year Mortality in the Cox Model of the Validation Cohort. (C) The Calibration Curves for

794 Predicting Mortality from the First Year to the Fifth Year of Follow-up in the Development 
795 Cohort. (D) The Calibration Curves for Predicting Mortality from the First Year to the Fifth Year

796 of Follow-up in the Validation Cohort.

797 Notes: Predicted 5-Year Mortality for each decile is the mean predicted risk score in each decile.

798 Error bars indicate standard deviations.

799

800

Figure 4 Decision Curve Analysis for C2ABS2-GLPK, GRACE, KAMIR and CAMI Scores.

801 (A) Decision Curve Analysis for C2ABS2-GLPK, GRACE, KAMIR and CAMI Scores in the

802 Development Cohort. (B) Decision Curve Analysis for C2ABS2-GLPK, GRACE, KAMIR and

803 CAMI Scores in the External Validation Cohorts.

804 Notes: Decision Curve Analysis for Most Discriminating Four Scores Applicable to More than $80550 \%$ of Validation Population (restricted cubic spline; imputed cohorts). Lines are Shown for

806 Standardised Net Benefit at Different Risk thresholds of Treating no Patients (black line) and 807 Treating All Patients (red line).

808 Abbreviations: GRACE: Global Registry of Acute Coronary Events; KAMIR: Korea Acute

809 Myocardial Infarction Registry; CAMI: China Acute Myocardial Infarction. 
810 Supplemental Figure Legends

\section{Figure S1 Variable Selection Using LASSO Regression.}

812 (A) LASSO Coefficient Profiles of the 45 Baseline Variables. (B) LASSO Regularization Level

813 was Chosen by 10-fold Cross-Validation Using the 1 Standard Error Rule.

814 Abbreviations: LASSO: least absolute shrinkage and selection operator.

815

816 Figure S2 Importance of Variables Selected by Random Forest Algorithm.

817 Abbreviations: MSE: mean squared error; STEMI: ST segment elevation myocardial infarction;

818 Door-to-Balloon time: Time from hospital arrival to first balloon inflation; WBC: white blood

819 cell; Hb: hemoglobin; ALT: alanine transaminase; Cr: creatinine; FBG: fast blood glucose; LDL-

820 C: low-density lipoprotein cholesterol; NT-proBNP: N-terminal pro-brain natriuretic peptide;

821 cTnI: cardiac Troponin I; LVEF: left ventricular ejection fraction; LA: left atrial; LVDd: left

822 ventricular end-diastolic diameter; RA: right atrial; Antiplatelets: aspirin, clopidogrel, ticagrelor;

823 Antihypertensives: angiotensin-converting enzyme inhibitor, angiotensin receptor blocker,

824 calcium-channel blocker, $\beta$-receptor blocker; PCI: percutaneous transluminal coronary

825 intervention.

826 
827 Figure S3 Subpopulation C-statistics Analysis for C2ABS2-GLPK, GRACE, KAMIR and 828 CAMI Scores.

829 (A) Subpopulation C-statistics Analysis for C2ABS2-GLPK, GRACE, KAMIR and CAMI

830 Scores in the Development Cohort. (B) Subpopulation C-statistics Analysis for C2ABS2-GLPK,

831 GRACE, KAMIR and CAMI Scores in the External Validation Cohort.

832 Notes: The Dashed Vertical Line Represents the C-statistics in the Total Population (0.80).

833 Horizontal Lines Represent 95\% CI.

834 Abbreviations: GRACE: Global Registry of Acute Coronary Events; KAMIR: Korea Acute

835 Myocardial Infarction Registry; CAMI: China Acute Myocardial Infarction. 
Figure 1

Flowchart for Patient Selection and Distribution of the Development and Validation Cohorts.

Abbreviations:AMI:acute myocardial infarction.

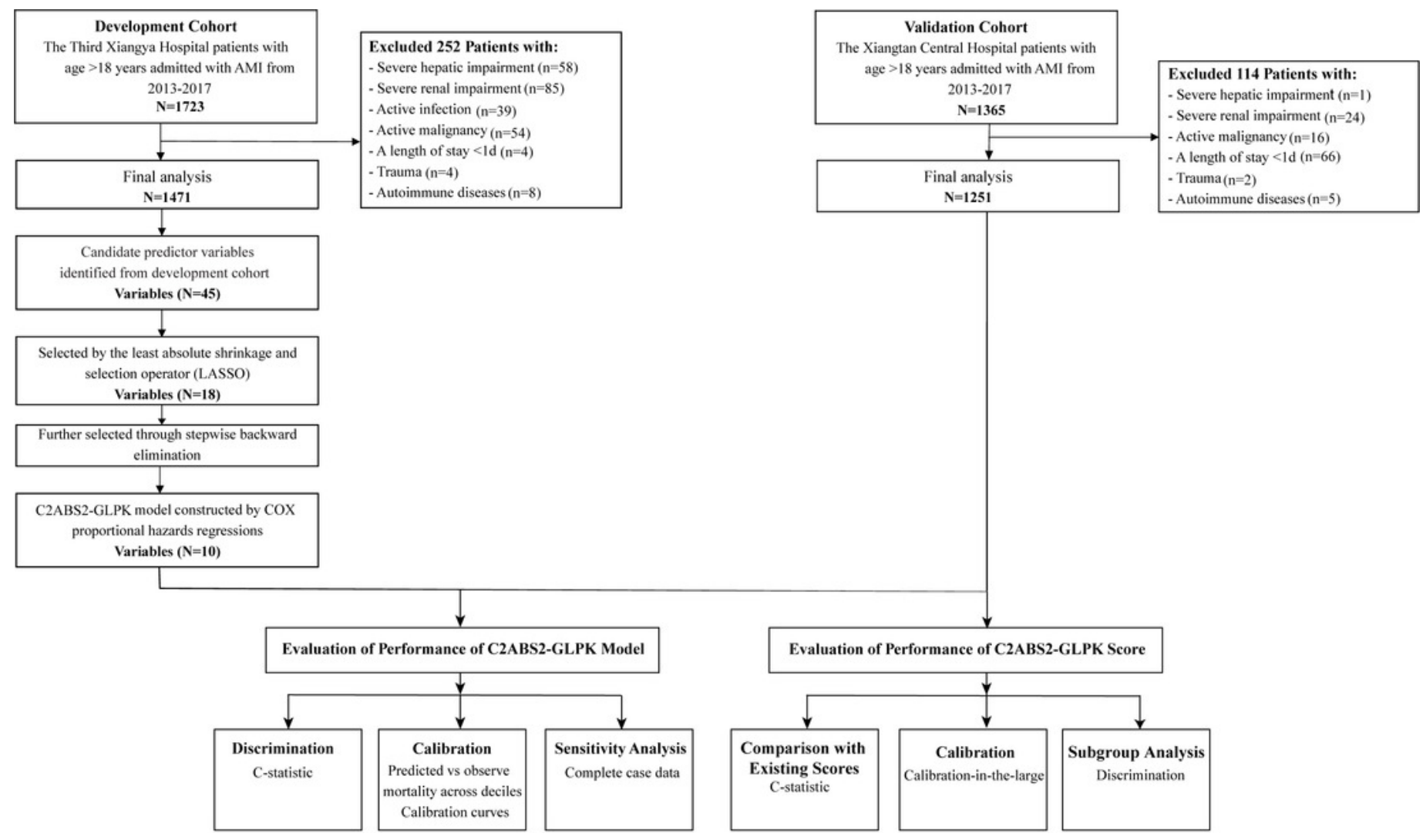




\section{Figure 2}

Nomogram for C2ABS2-GLPK Score to Predict 1-, 2-, 3-, 4-, 5-Year All-Cause Mortality in Patients with AMI and Kaplan-Meier Survival Curves Stratified by C2ABS2-GLPK Score.

(A) Nomogram for Predicting 1-, 2-, 3-, 4-, 5-Year All-Cause Mortality among Patients with AMI. (B) Kaplan-Meier Survival Curves for AMI Patients with Risk Stratified by C2ABS2-GLPK Score according to the Xtile Analysis in the Development Cohort. (C) Kaplan-Meier Survival Curves for AMI Patients with Risk Stratified by C2ABS2-GLPK Score according to the X-tile Analysis in the External Validation Cohort.

Notes: Nomogram for the prediction of 1-, 2-, 3-, 4-, 5-Year All-cause Mortality-Free in Patients with AMI. The patient's age is located on the row labeled "Age, $y$ ", the patient's left ventricular end-diastolic diameter is located on the row labeled "Left Ventricular end-diastolic Diameter, $\mathrm{mm}$ ", the patient's fasting blood glucose level is located on the row labeled "Fasting Blood Glucose, $\mathrm{mmol} / \mathrm{L}$ ", the patient's creatinine level is located on the row labeled "Creatinine, $\mu \mathrm{mol} / \mathrm{L}$ ", the patient's NT-proBNP level is located on the row labeled "NT-proBNP, pg/ml" , the patient's Killip classification is located on the row labeled "Killip, classifications" , and a straight line is drawn up to the row labeled "Points" to determine the corresponding points. This process is repeated for each of the remaining factors by drawing a straight line to the "Points" row to determine the points associated with each factor. After summing the total points, one locates the appropriate total point number and draws a straight line from this to the rows labeled "Predicted 1-, 2-, 3-, 4-, 5-Year All-cause Mortality-Free probability to determine the patient's predicted survival probability.

Abbreviations: AMI: acute myocardial infarction; NT-proBNP: N-terminal pro-brain natriuretic peptide; PCl: percutaneous transluminal coronary intervention. 
A

Points

Age, years

NT-proBNP, pg/mL

Fasting Blood Glucose, mmol/L

$\mathrm{PCl}$

Killip, classifications

Stroke

Cardiac Arrest

Left Ventricular end-Diastolic Diameter,mm

Total Points

1-Year All-cause Mortality-Free Probability

2-Year All-cause Mortality-Free Probability

3-Year All-cause Mortality-Free Probability

4-Year All-cause Mortality-Free Probability

5-Year All-cause Mortality-Free Probability

Risk Stratifications

B

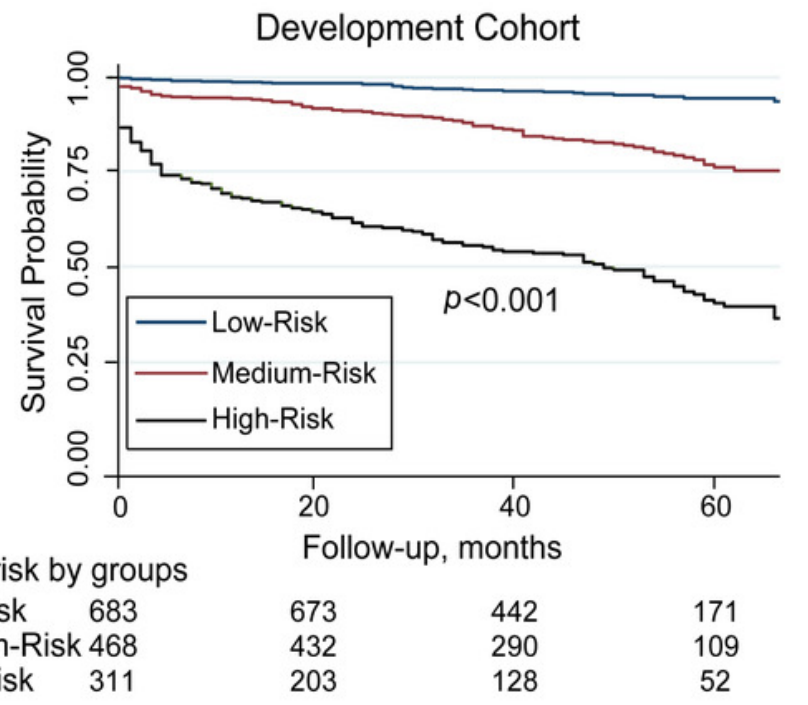

$0-55$

Medium-Risk 468

High-Risk 311

203
52
Creatinine, $\mu \mathrm{mol} / \mathrm{L}$

Statins Therapy

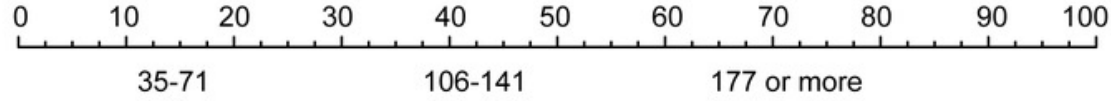

\begin{tabular}{|c|c|c|c|}
\hline $0-35$ & $40-49^{71-106}$ & $141-17760-69$ & $80-89$ \\
\hline $0-40$ & & & \\
\hline
\end{tabular}

$\begin{array}{r}450-900 \\ 0-450 \quad 900-1800 \\ \hline\end{array}$
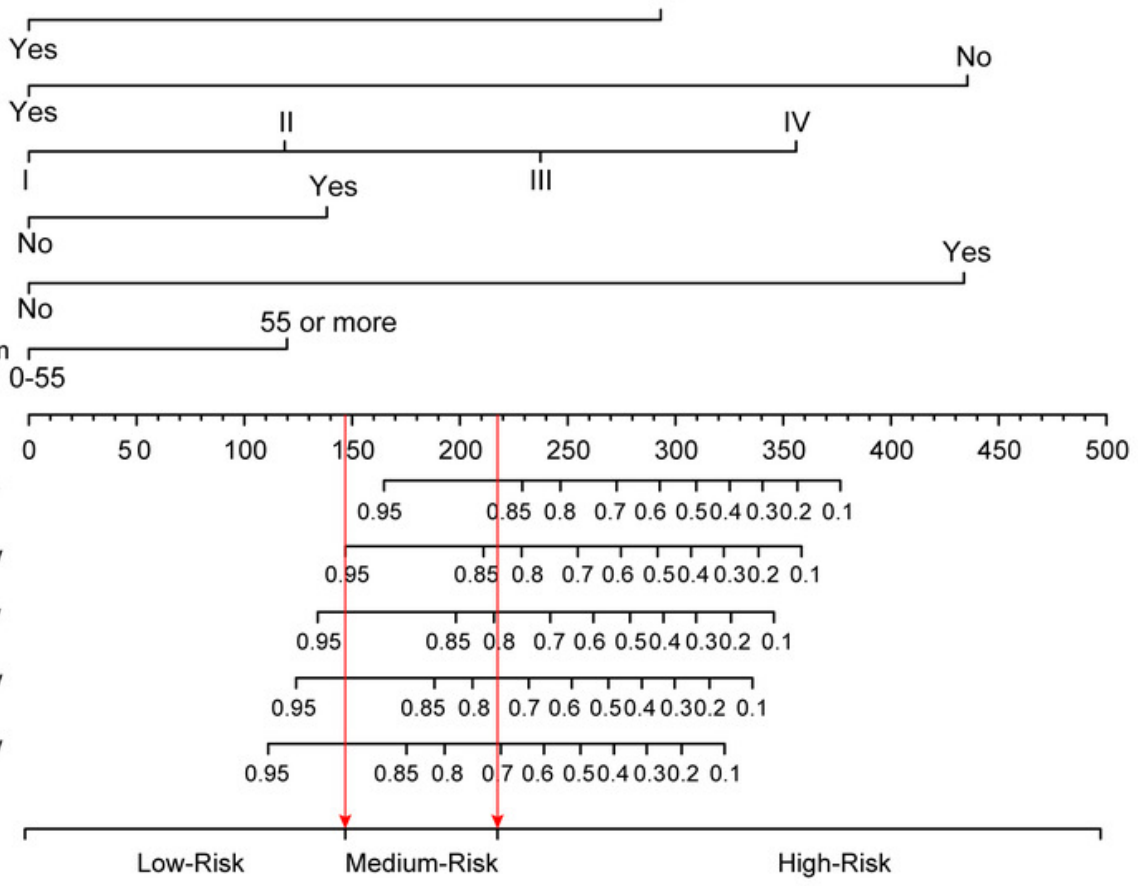

C

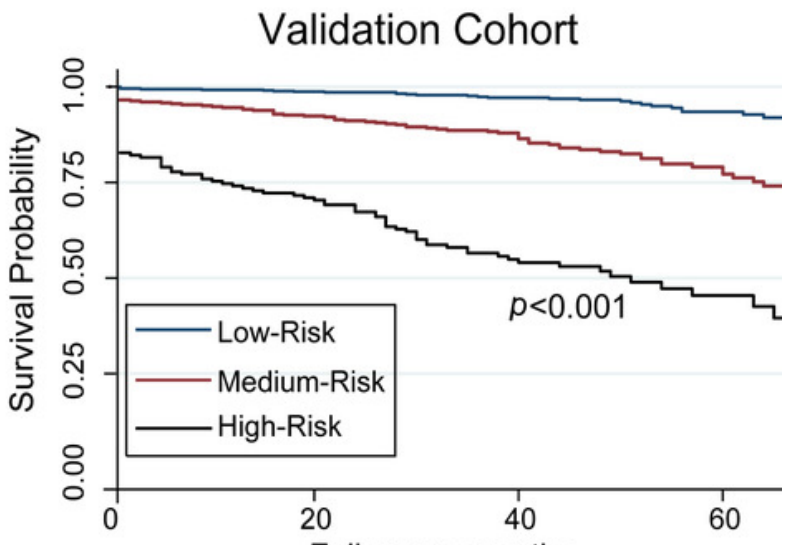

No. at risk by groups

Low-Risk 620

Medium-Risk 404

Follow-up, months

No. at risk by groups
High-Risk 162
612

115
396

236

66
145

21 


\title{
Figure 3
}

\section{Calibration of C2ABS2-GLPK Score in the Development Cohort and Validation Cohort.}

\begin{abstract}
(A) Agreement between the Deciles of Observed vs Predicted 5-Year Mortality in the Cox Model of the Development Cohort. (B) Agreement between the Deciles of Observed vs Predicted 5-Year Mortality in the Cox Model of the Validation Cohort. (C) The Calibration Curves for Predicting Mortality from the First Year to the Fifth Year of Follow-up in the Development Cohort. (D) The Calibration Curves for Predicting Mortality from the First Year to the Fifth Year of Follow-up in the Validation Cohort.
\end{abstract}

Notes: Predicted 5-Year Mortality for each decile is the mean predicted risk score in each decile. Error bars indicate standard deviations. 
A

Development Cohort

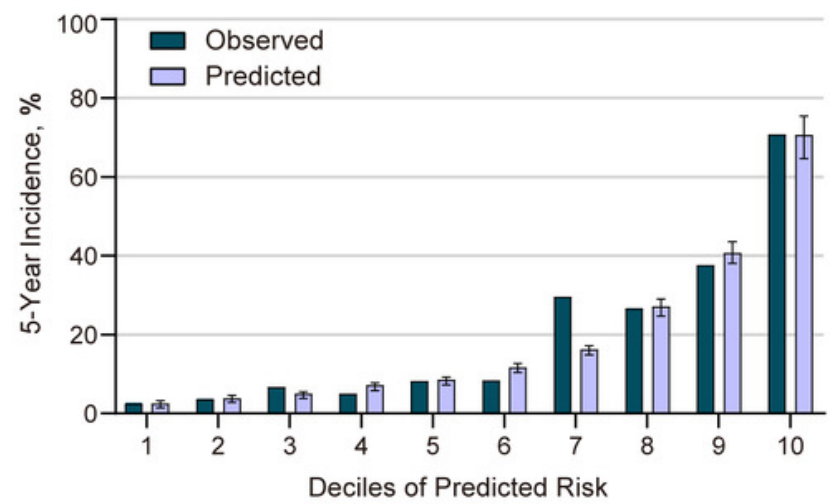

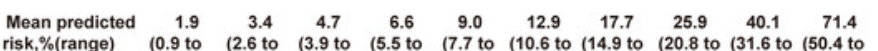
$\begin{array}{rrrrrrrr}\text { risk,\%(range) } & (0.9 \text { to } & (2.6 \text { to } & (3.9 \text { to } & (5.5 \text { to } & (7.7 \text { to } & (10.6 \text { to }(14.9 \text { to } & (20.8 \text { to }(31.6 \text { to }(50.4 \text { to } \\ <2.6) & <3.9) & <5.5) & <7.7)<10.6)<14.9)<20.8)<31.6)<50.3)<99.9)\end{array}$ $\begin{array}{lrrrrrrrrrr}\text { No.of events } & 3 & 5 & 10 & 7 & 12 & 13 & 42 & 37 & 57 & 106 \\ \text { No.of participants } & 147 & 147 & 147 & 147 & 147 & 147 & 147 & 147 & 147 & 148\end{array}$
B

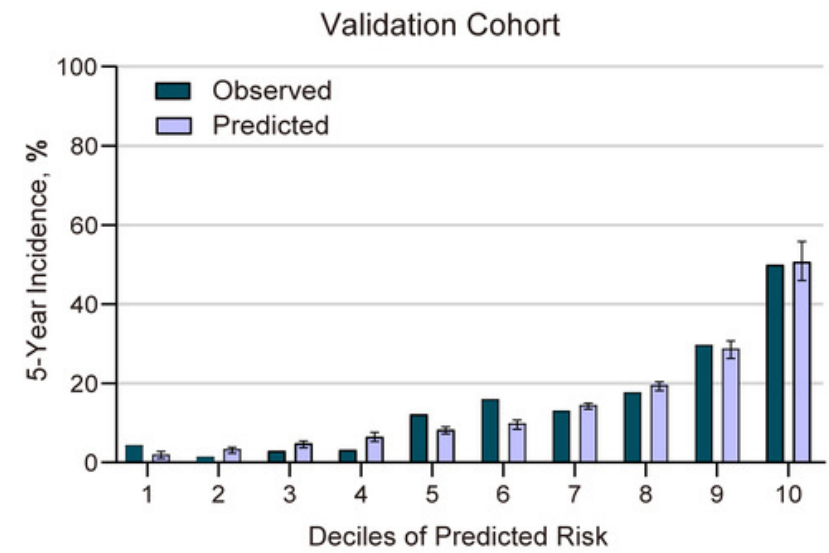

C

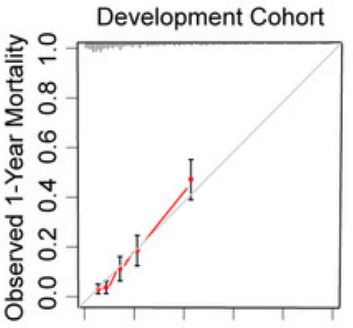

$\begin{array}{llllll}0.0 & 0.2 & 0.4 & 0.6 & 0.8 & 1.0\end{array}$

Predicted 1-Year Mortality

D

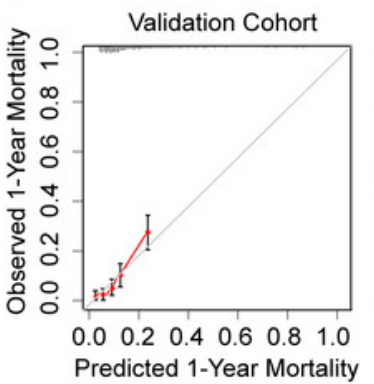

Development Cohort

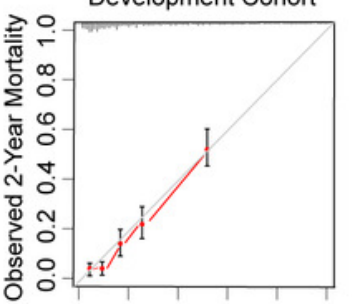

$\begin{array}{llllll}0.0 & 0.2 & 0.4 & 0.6 & 0.8 & 1.0\end{array}$

Predicted 2-Year Mortality

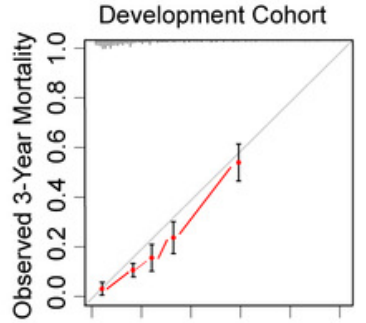

$\begin{array}{llllll}0.0 & 0.2 & 0.4 & 0.6 & 0.8 & 1.0\end{array}$

Predicted 3-Year Mortality

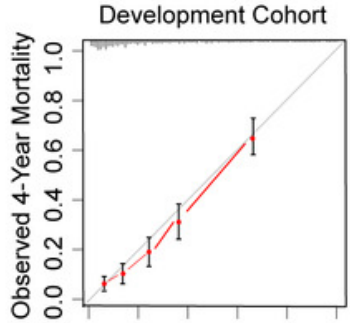

$\begin{array}{llllll}0.0 & 0.2 & 0.4 & 0.6 & 0.8 & 1.0\end{array}$

Predicted 4-Year Mortality

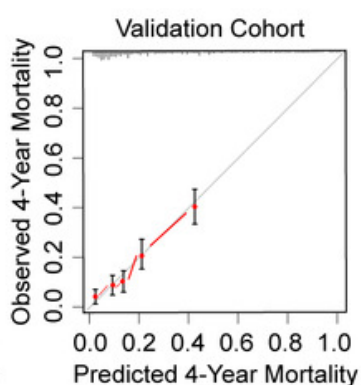

Development Cohort
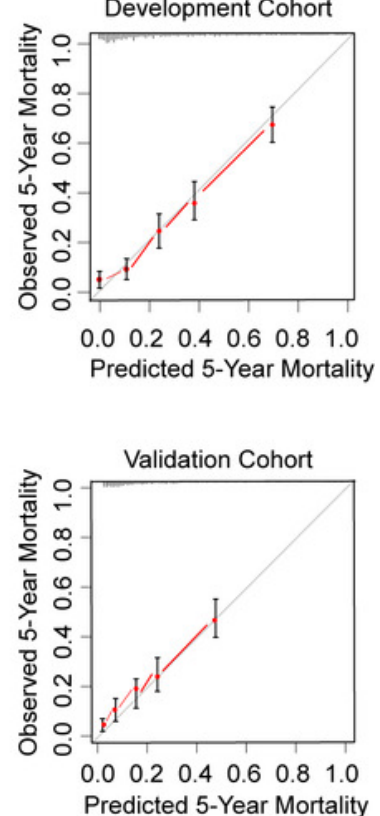
Figure 4

Decision Curve Analysis for C2ABS2-GLPK, GRACE, KAMIR and CAMI Scores.

(A) Decision Curve Analysis for C2ABS2-GLPK, GRACE, KAMIR and CAMI Scores in the Development Cohort. (B) Decision Curve Analysis for C2ABS2-GLPK, GRACE, KAMIR and CAMI Scores in the External Validation Cohorts.

Notes: Decision Curve Analysis for Most Discriminating Four Scores Applicable to More than $50 \%$ of Validation Population (restricted cubic spline; imputed cohorts). Lines are Shown for Standardised Net Benefit at Different Risk thresholds of Treating no Patients (black line) and Treating All Patients (red line).

Abbreviations: GRACE: Global Registry of Acute Coronary Events; KAMIR: Korea Acute Myocardial Infarction Registry; CAMI: China Acute Myocardial Infarction.

A

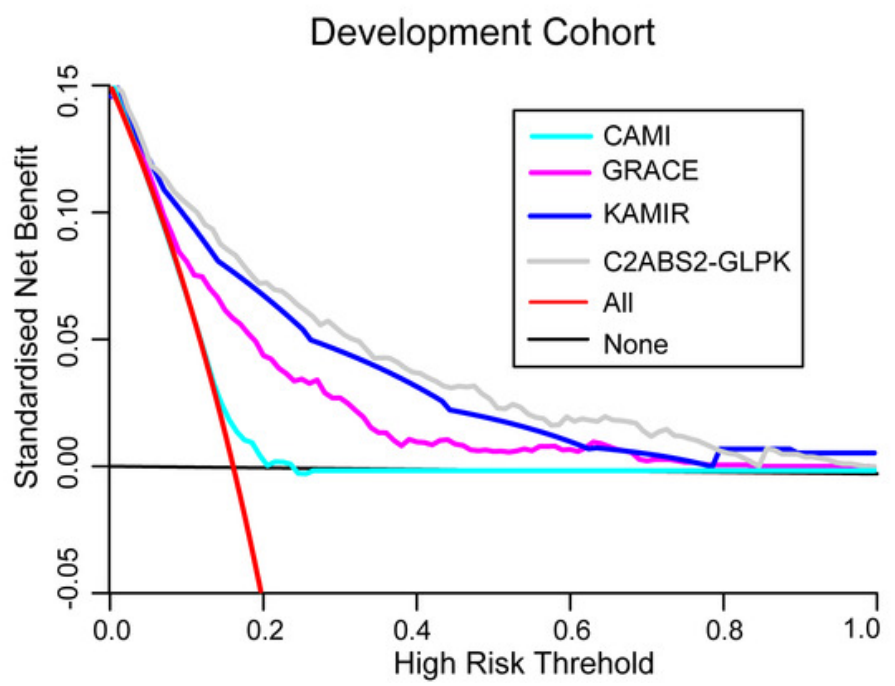

B

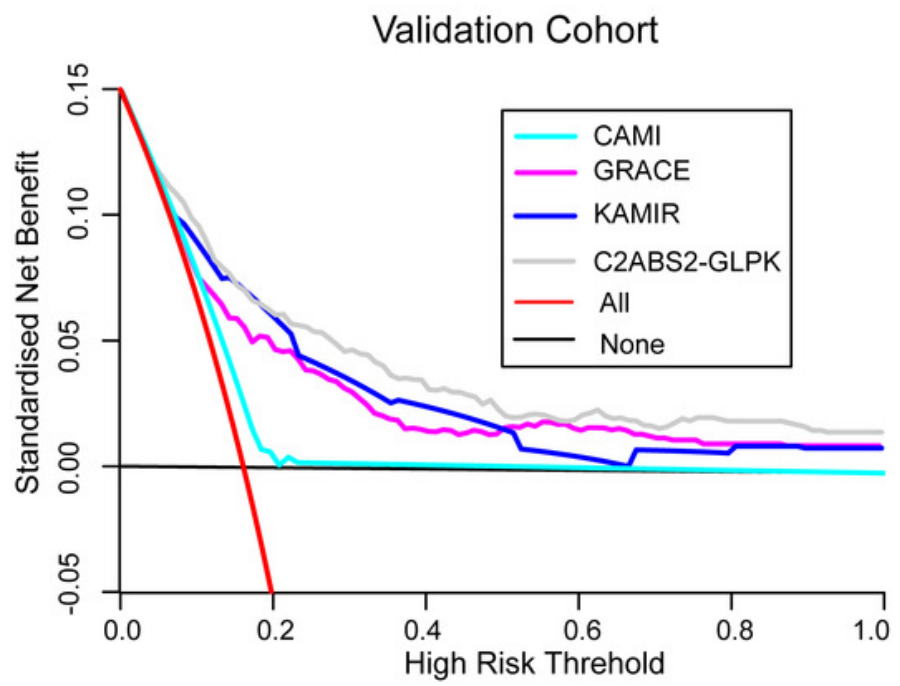




\section{Table $\mathbf{1}$ (on next page)}

Baseline Characteristics of Patients with AMI in the Model Development and Validation Database.

Abbreviations: AMI: acute myocardial infarction; IQR: interquartile range; BMI: body mass index; STEMI: ST segment elevation myocardial infarction; Door-to-Balloon time: Time from hospital arrival to first balloon inflation; HR: heart rate; WBC: white blood cell; $\mathrm{Hb}$ : hemoglobin; PLT: platelet; ALT: alanine transaminase; Cr: creatinine; FBG: fast blood glucose; LDL-C: low-density lipoprotein cholesterol; NT-proBNP: N-terminal pro-brain natriuretic peptide; cTnl: cardiac Troponin I; MI: myocardial infarction; $\mathrm{PCl}$ : percutaneous transluminal coronary intervention; AF: atrial fibrillation; LVEF: left ventricular ejection fraction; LA: left atrial; LVDd: left ventricular end-diastolic diameter; RA: right atrial; RV: right ventricular; LV: left ventricular; Antiplatelets: aspirin, clopidogrel, ticagrelor; Antihypertensives: angiotensinconverting enzyme inhibitor, angiotensin receptor blocker, calcium-channel blocker, $\beta$ receptor blocker; CABG: coronary artery bypass grafting. 
1 Table 1. Baseline Characteristics of Patients with AMI in the Model Development and Validation Database.

\begin{tabular}{|c|c|c|c|c|c|c|c|c|}
\hline \multirow[t]{2}{*}{ Variables } & \multicolumn{4}{|c|}{ Development database } & \multicolumn{4}{|c|}{ Validation database } \\
\hline & $\begin{array}{l}\text { Total } \\
(\mathrm{N}=1471)\end{array}$ & $\begin{array}{l}\text { Survivors } \\
(\mathrm{N}=1170)\end{array}$ & $\begin{array}{l}\text { Non-survivors } \\
(\mathrm{N}=\mathbf{2 9 2})\end{array}$ & $\begin{array}{l}\text { Censor } \\
(\mathrm{N}=9)\end{array}$ & $\begin{array}{l}\text { Total } \\
(\mathrm{N}=1251)\end{array}$ & $\begin{array}{l}\text { Survivors } \\
(\mathrm{N}=1000)\end{array}$ & $\begin{array}{l}\text { Non-survivors } \\
(\mathrm{N}=\mathbf{1 8 6})\end{array}$ & $\begin{array}{l}\text { Censor } \\
(N=65)\end{array}$ \\
\hline Age, median (IQR), y & $64(55-72)$ & $62(53-70)$ & $71(63-77)$ & $72(68-76)$ & $65(56-73)$ & $63(54-71)$ & $72(66-78)$ & $66(59-72)$ \\
\hline Male, n (\%) & $1050(71.4)$ & $860(73.5)$ & $187(64.0)$ & $3(33.3)$ & $911(72.8)$ & $751(75.2)$ & $113(60.8)$ & $47(72.3)$ \\
\hline $\begin{array}{l}\text { BMI, median (IQR), } \\
\mathrm{kg} / \mathrm{m}^{2}\end{array}$ & $\begin{array}{l}23.9 \\
(21.8-26.0)\end{array}$ & $\begin{array}{l}24.0 \\
26.0)\end{array}$ & $\begin{array}{l}23.8 \\
(21.4-25.7)\end{array}$ & $\begin{array}{l}21.9 \\
(20.7-23.3)\end{array}$ & $\begin{array}{l}23.4 \\
24.8)\end{array}$ & $\begin{array}{l}23.4 \\
(22.2-24.9)\end{array}$ & $\begin{array}{l}23.4 \\
(22.0-24.0)\end{array}$ & $\begin{array}{l}23.4 \\
24.0)\end{array}$ \\
\hline $\begin{array}{l}\text { Current smoking, } \mathrm{n} \\
(\%)\end{array}$ & $156(10.6)$ & $126(10.8)$ & $29(9.9)$ & $1(11.1)$ & $80(6.4)$ & $67(6.7)$ & $10(5.4)$ & $3(4.6)$ \\
\hline $\begin{array}{l}\text { ST } \\
\text { depression, } \mathrm{n}(\%)\end{array}$ & $497(33.8)$ & $388(33.2)$ & 107 (36.6) & $2(22.2)$ & $256(20.5)$ & $196(19.6)$ & $47(25.3)$ & $13(20.0)$ \\
\hline STEMI, n (\%) & $922(62.7)$ & $757(64.7)$ & $159(54.5)$ & $6(66.7)$ & $919(73.5)$ & $764(76.4)$ & $108(58.1)$ & $47(72.3)$ \\
\hline $\begin{array}{l}\text { Acute Anterior MI, n } \\
(\%)\end{array}$ & $494(33.5)$ & $401(34.3)$ & $77(26.4)$ & $6(66.7)$ & $451(36.1)$ & $373(37.3)$ & $58(31.2)$ & $20(30.8)$ \\
\hline $\begin{array}{l}\text { Left main coronary } \\
\text { lesion, } \mathrm{n}(\%)\end{array}$ & $107(7.3)$ & $85(7.3)$ & $22(7.5)$ & $0(0.0)$ & $114(9.1)$ & $89(8.9)$ & $21(11.3)$ & $4(6.2)$ \\
\hline $\begin{array}{l}\text { Coronary multivessel } \\
\text { lesion, } \mathrm{n}(\%)\end{array}$ & $515(35.0)$ & $417(35.6)$ & $94(32.2)$ & $4(44.4)$ & $34(2.7)$ & $22(2.2)$ & $8(4.3)$ & $4(6.2)$ \\
\hline \multicolumn{9}{|c|}{ Door-to-Balloon time, $\mathrm{n}(\%), \mathrm{h}$} \\
\hline$\leq 4$ & $191(13.0)$ & $146(12.5)$ & $43(14.7)$ & $2(22.2)$ & $391(31.3)$ & $315(31.5)$ & $65(34.9)$ & $11(16.9)$ \\
\hline$>4$ & $1280(87.0)$ & $1024(87.5)$ & $249(85.3)$ & $7(77.8)$ & $857(68.5)$ & $701(70.1)$ & $111(59.7)$ & $45(69.2)$ \\
\hline
\end{tabular}

Symptoms, n (\%) 
Cardiac Arrest

$$
35(2.4) \quad 11(0.9) \quad 24(8.2)
$$

$$
0(0.0)
$$

$15(1.5)$

$20(10.8)$

$3(4.6)$

Killip Classification, $\mathrm{n}(\%)$

$\begin{array}{lllllllll}\text { I } & 1028(69.9) & 890(76.1) & 133(45.5) & 5(55.6) & 698(55.8) & 597(59.7) & 63(29.8) & 38(58.5) \\ \text { II } & 274(18.6) & 199(17.0) & 73(25.0) & 2(22.2) & 404(32.3) & 321(32.1) & 66(35.5) & 17(26.2) \\ \text { III } & 82(5.6) & 43(3.7) & 37(12.7) & 2(22.2) & 83(6.6) & 48(4.8) & 29(15.6) & 6(9.2) \\ \text { IV } & 87(5.9) & 38(3.2) & 49(16.8) & 0(0.0) & 66(5.3) & 34(3.4) & 28(15.1) & 4(6.2)\end{array}$

Signs, median (IQR)

HR, beats/min

$75(66-87)$

$74(66-85) \quad 80(70-92)$

$86(76-92)$

$78(68-88)$

$78(68-87)$

\begin{tabular}{|c|c|c|c|c|c|c|c|c|}
\hline Systolic & $\begin{array}{l}128 \\
(111-142)\end{array}$ & $\begin{array}{l}128 \\
142)\end{array}$ & $\begin{array}{l}128 \\
(110-143)\end{array}$ & $\begin{array}{l}124 \\
126)\end{array}$ & $\begin{array}{l}130 \\
149)\end{array}$ & $\begin{array}{l}130 \\
(120-150)\end{array}$ & $\begin{array}{l}130 \\
(115-146)\end{array}$ & $\begin{array}{l}130 \\
(120-143)\end{array}$ \\
\hline Diastolic & $76(68-84)$ & $76(69-84)$ & $76(66-84)$ & $76(74-80)$ & $80(70-90)$ & $80(70-90)$ & $80(70-90)$ & $80(75-90)$ \\
\hline \multicolumn{9}{|c|}{ Laboratory Findings, median (IQR) } \\
\hline $\mathrm{WBC},{ }^{*} 10^{9} / \mathrm{L}$ & $\begin{array}{l}9.0 \\
(7.1-11.4)\end{array}$ & $\begin{array}{l}9.0 \\
(7.2-11.3)\end{array}$ & $\begin{array}{l}9.1 \\
(6.8-12.0)\end{array}$ & $\begin{array}{l}9.5 \\
10.6)\end{array}$ & $\begin{array}{l}9.4 \\
11.9)\end{array}$ & $\begin{array}{l}9.4 \\
(7.5-11.8)\end{array}$ & $\begin{array}{l}9.4 \\
(7.5-12.1)\end{array}$ & $\begin{array}{l}9.7 \\
(6.8-12.4)\end{array}$ \\
\hline $\mathrm{Hb}, \mathrm{g} / \mathrm{L}$ & $\begin{array}{l}130 \\
(117-144)\end{array}$ & $\begin{array}{l}133 \\
145)\end{array}$ & $\begin{array}{l}122 \\
(109-133)\end{array}$ & $\begin{array}{l}121 \\
130)\end{array}$ & $\begin{array}{l}130 \\
142)\end{array}$ & $\begin{array}{l}131 \\
(119-142)\end{array}$ & $\begin{array}{l}127 \\
(116-141)\end{array}$ & $\begin{array}{l}133 \\
(120-141)\end{array}$ \\
\hline $\mathrm{PLT}, * 10^{9} / \mathrm{L}$ & $\begin{array}{l}203 \\
(166-246)\end{array}$ & $\begin{array}{l}205 \\
249)\end{array}$ & $\begin{array}{l}194 \\
(155-235)\end{array}$ & $\begin{array}{l}164 \\
252)\end{array}$ & $\begin{array}{l}189 \\
226)\end{array}$ & $\begin{array}{l}190 \\
(153-227)\end{array}$ & $\begin{array}{l}189 \\
(155-232)\end{array}$ & $\begin{array}{l}188 \\
(149-219)\end{array}$ \\
\hline ALT, U/L & $36(23-54)$ & $37(24-54)$ & $33(20-57)$ & $33(31-40)$ & $34(20-49)$ & $34(19-48)$ & $35(20-52)$ & $36(23-51)$ \\
\hline $\mathrm{Cr}, \mu \mathrm{mol} / \mathrm{L}$ & $82(68-99)$ & $80(68-94)$ & $93(75-126)$ & $79(65-101)$ & $79(67-95)$ & $78(66-93)$ & $87(72-112)$ & $75(65-82)$ \\
\hline
\end{tabular}

$89(70-95)$

$80(73-90)$

Blood Pressure, median (IQR), mm Hg 


$\begin{array}{lllllllll}\text { FBG, } \mathrm{mmol} / \mathrm{L} & 5.9(5.1-7.2) & 5.9(5.0-7.1) & 6.4(5.2-8.1) & 4.5(4.2-8.3) & 5.9(5.0-7.3) & 5.9(5.0-7.1) & 6.2(5.1-8.1) & 6.3(5.1-6.9) \\ \text { LDL-C, } \mathrm{mmol} / \mathrm{L} & 2.5(1.9-3.0) & 2.5(2.0-3.0) & 2.4(1.9-2.9) & 2.3(2.0-2.6) & 2.8(2.2-3.3) & 2.8(2.2-3.3) & 2.7(2.2-3.2) & 3.0(2.4-3.5)\end{array}$

Biomarkers cardiac injury, median (IQR)

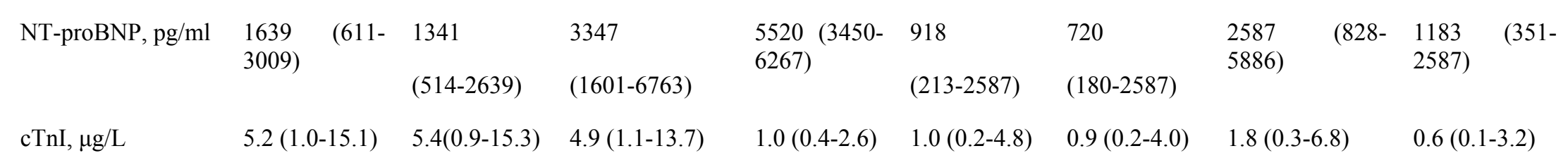

Prior History, n (\%)

$\begin{array}{lllllllll}\text { Prior MI } & 28(1.9) & 21(1.8) & 6(2.1) & 1(11.1) & 11(0.9) & 7(0.7) & 3(1.6) & 1(1.5) \\ \text { Prior PCI } & 22(1.5) & 16(1.4) & 5(1.7) & 1(11.1) & 90(7.2) & 71(7.1) & 9(4.8) & 10(15.4) \\ \text { Comorbidities, n (\%) } & & & & & & & & \\ \text { Hypertension } & 828(56.3) & 640(54.7) & 182(62.3) & 6(66.7) & 703(56.2) & 574(57.4) & 112(60.2) & 39(60.0) \\ \text { Diabetes } & 478(32.5) & 369(31.5) & 104(35.6) & 5(55.6) & 218(17.4) & 162(16.2) & 40(21.5) & 16(24.6) \\ \text { Stroke } & 173(11.8) & 111(9.5) & 60(20.5) & 2(22.2) & 127(10.2) & 97(9.7) & 25(13.4) & 5(7.7) \\ \text { AF } & 23(1.6) & 14(1.2) & 9(3.0) & 0(0.0) & 73(5.8) & 55(5.5) & 13(7.0) & 5(7.7)\end{array}$

Echocardiography

\begin{tabular}{|c|c|c|c|c|c|c|c|c|}
\hline $\begin{array}{l}\text { LVEF, median } \\
\text { IQR), \% }\end{array}$ & $54(49-61)$ & $54(50-62)$ & $54(44-54)$ & $46(30-54)$ & $51(44-58)$ & $51(45-59)$ & $50(39-56)$ & $50(44-56)$ \\
\hline $\begin{array}{l}\mathrm{LA}, \text { median (IQR), } \\
\mathrm{mm}\end{array}$ & $33(31-35)$ & $33(30-35)$ & $33(33-36)$ & $34(28-38)$ & $35(32-37)$ & $35(32-37)$ & $35(33-37)$ & $35(32-37)$ \\
\hline $\begin{array}{l}\text { LVDd, median } \\
\text { (IQR), mm }\end{array}$ & $49(45-51)$ & $49(45-50)$ & $49(48-53)$ & $49(45-55)$ & $49(46-51)$ & $49(45-51)$ & $49(47-55)$ & $49(46-53)$ \\
\hline $\begin{array}{l}\text { RA, median (IQR), } \\
\text { mm }\end{array}$ & $30(29-32)$ & $30(28-32)$ & $30(30-33)$ & $30(27-31)$ & $35(33-36)$ & $35(33-36)$ & $35(34-36)$ & $35(33-37)$ \\
\hline
\end{tabular}




\begin{tabular}{|c|c|c|c|c|c|c|c|c|}
\hline $\begin{array}{l}\mathrm{RV} \text {, median (IQR), } \\
\mathrm{mm}\end{array}$ & $29(27-31)$ & $29(27-31)$ & $29(28-31)$ & $29(28-30)$ & $19(18-19)$ & $19(18-19)$ & $19(18-19)$ & $19(17-19)$ \\
\hline $\begin{array}{l}\text { Aortic } \\
\text { Regurgitation, } \mathrm{n}(\%)\end{array}$ & $365(24.8)$ & $262(23.0)$ & $100(34.2)$ & $3(33.3)$ & $551(44.1)$ & $418(42.2)$ & $106(57.0)$ & $27(41.5)$ \\
\hline $\begin{array}{l}\text { Mitral } \\
\text { Regurgitation, n (\%) }\end{array}$ & $978(66.5)$ & $739(64.0)$ & $233(79.8)$ & $6(66.7)$ & $970(77.5)$ & $780(78.0)$ & $144(77.4)$ & $46(70.8)$ \\
\hline $\begin{array}{l}\text { Tricuspid } \\
\text { Regurgitation, } \mathrm{n}(\%)\end{array}$ & $555(37.7)$ & $433(37.1)$ & $118(40.4)$ & $4(44.4)$ & $910(72.7)$ & $722(72.2)$ & $144(77.4)$ & $44(67.7)$ \\
\hline $\begin{array}{l}\text { Pulmonary } \\
\text { Regurgitation, n (\%) }\end{array}$ & $220(15.0)$ & $171(15.1)$ & $47(16.1)$ & $2(22.2)$ & $30(2.4)$ & $27(2.7)$ & $2(1.1)$ & $1(1.5)$ \\
\hline $\begin{array}{l}\text { Decreased Left } \\
\text { Ventricular } \\
\text { Compliance, n (\%) }\end{array}$ & $473(32.2)$ & $417(35.3)$ & $54(18.5)$ & $2(22.2)$ & $1144(91.4)$ & $916(91.6)$ & $170(91.4)$ & $58(89.2)$ \\
\hline \multicolumn{9}{|c|}{ Baseline medications, $\mathrm{n}(\%)$} \\
\hline Antiplatelets & $1461(99.3)$ & $1168(99.8)$ & $284(97.3)$ & $9(100.0)$ & $1232(98.5)$ & $984(98.4)$ & $183(98.4)$ & $65(100.0)$ \\
\hline Statins & $1457(99.0)$ & $1166(99.6)$ & $282(96.6)$ & $9(100.0)$ & $1251(100.0)$ & $1000(100.0)$ & $186(100.0)$ & $65(100.0)$ \\
\hline Antihypertensives & $1354(92.1)$ & $1106(94.0)$ & $239(81.8)$ & $9(100.0)$ & $1096(87.6)$ & $873(87.3)$ & $161(86.6)$ & $62(95.4)$ \\
\hline PCI & $844(57.4)$ & $760(65.0)$ & $81(27.7)$ & $3(33.3)$ & $867(69.3)$ & 719 (71.9) & $99(53.2)$ & $49(75.4)$ \\
\hline CABG & $2(0.1)$ & $2(0.2)$ & $0(0.0)$ & $0(0.0)$ & $1(0.1)$ & $0(0.0)$ & $1(0.5)$ & $0(0.0)$ \\
\hline
\end{tabular}

2 Abbreviations: AMI: acute myocardial infarction; IQR: interquartile range; BMI: body mass index; STEMI: ST segment elevation myocardial infarction; Doorto-Balloon time: Time from hospital arrival to first balloon inflation; HR: heart rate; WBC: white blood cell; Hb: hemoglobin; PLT: platelet; ALT: alanine transaminase; Cr: creatinine; FBG: fast blood glucose; LDL-C: low-density lipoprotein cholesterol; NT-proBNP: N-terminal pro-brain natriuretic peptide; cTnI: cardiac Troponin I; MI: myocardial infarction; PCI: percutaneous transluminal coronary intervention; AF: atrial fibrillation; LVEF: left ventricular ejection fraction; LA: left atrial; LVDd: left ventricular end-diastolic diameter; RA: right atrial; RV: right ventricular; LV: left ventricular; Antiplatelets: aspirin,

7 clopidogrel, ticagrelor; Antihypertensives: angiotensin-converting enzyme inhibitor, angiotensin receptor blocker, calcium-channel blocker, $\beta$-receptor blocker;

8 CABG: coronary artery bypass grafting. 


\section{Table 2 (on next page)}

Selected Variables and Cox Model for Predicting 5-year Morality in the Development Cohort.

Abbreviations: Cl: confidence intervals; FBG: fast blood glucose; LVDd: left ventricular enddiastolic diameter; Cr: Creatinine; NT-proBNP: N-terminal pro-brain natriuretic peptide; PCl: percutaneous transluminal coronary intervention. 
1 Table 2 Selected Variables and Cox Model for Predicting 5-year Morality in the 2 Development Cohort.

\begin{tabular}{|c|c|c|}
\hline Variables & Hazard Ratio (95\%CI) & $p$ value \\
\hline \multicolumn{3}{|c|}{ Cardiac Arrest } \\
\hline No & 1.0 (reference) & \\
\hline Yes & $5.0(3.1-8.2)$ & $<0.001$ \\
\hline \multicolumn{3}{|l|}{ Stroke } \\
\hline No & 1.0 (reference) & \\
\hline Yes & $1.7(1.2-2.3)$ & 0.001 \\
\hline \multicolumn{3}{|c|}{ Killip, classifications } \\
\hline I & 1.0 (reference) & \\
\hline II & $1.3(1.0-1.9)$ & 0.034 \\
\hline III & $2.5(1.7-3.6)$ & $<0.001$ \\
\hline IV & $3.9(2.7-5.6)$ & $<0.001$ \\
\hline \multicolumn{3}{|c|}{ FBG, $\mathbf{m m o l} / \mathbf{L}$} \\
\hline $0-10$ & 1.0 (reference) & \\
\hline$\geq 10$ & $1.5(1.1-2.2)$ & 0.016 \\
\hline \multicolumn{3}{|c|}{ LVDd, mm } \\
\hline $0-60$ & 1.0 (reference) & \\
\hline$\geq 60$ & $1.9(1.3-2.8)$ & 0.002 \\
\hline \multicolumn{3}{|l|}{ Age, years } \\
\hline$<40$ & 1.0 (reference) & \\
\hline $40-49$ & $2.0(0.3-16.5)$ & 0.506 \\
\hline $50-59$ & $3.1(0.4-23.5)$ & 0.268 \\
\hline $60-69$ & $6.0(0.8-44.1)$ & 0.080 \\
\hline $70-79$ & $7.5(1.0-55.5)$ & 0.048 \\
\hline $80-89$ & $11.1(1.5-82.9)$ & 0.019 \\
\hline
\end{tabular}




$\begin{array}{llc}0-35 & 14.5(1.9-110.4) & 0.010 \\ 36-70 & 1.0 \text { (reference) } & \\ 71-105 & 1.2(0.9-1.6) & 0.289 \\ 106-140 & 1.5(1.0-2.2) & 0.035 \\ 141-176 & 2.4(1.5-3.8) & <0.001 \\ \geq 177 & 2.3(1.4-4.1) & 0.002\end{array}$

NT-ProBNP, pg/ml

0-300 1.0 (reference)

$\begin{array}{lll}\geq 300 & 2.0(1.1-3.5) & 0.024\end{array}$

Statins therapy

No 1.0 (reference)

Yes $\quad 0.2(0.1-0.5) \quad<0.001$

PCI

No $\quad 1.0$ (reference)

$\begin{array}{lll}\text { Yes } & 0.4(0.3-0.5) & <0.001\end{array}$

3 Abbreviations: CI: confidence intervals; FBG: fast blood glucose; LVDd: left ventricular end-diastolic diameter; Cr:

4 Creatinine; NT-proBNP: N-terminal pro-brain natriuretic peptide; PCI: percutaneous transluminal coronary 5 intervention. 